\title{
Impedance Modeling for Mixed Conductors with Simultaneous Insertion \& Electrocatalytic Reactions: A Case Study of Transition-Metal Hydroxides in Aqueous Electrolyte
}

\author{
Allen Yu-Lun Liang ${ }^{1,3, z} \&$ William C. Chueh ${ }^{2,3}$ \\ ${ }^{1}$ Department of Chemistry, Stanford University, Stanford, CA 94305 \\ ${ }^{2}$ Department of Materials Science and Engineering, Stanford University, Stanford, CA 94305 \\ ${ }^{3}$ Stanford Institute for Materials and Energy Science, SLAC National Accelerator Laboratory, \\ Menlo Park, CA 94025 \\ ${ }^{\text {z }}$ Corresponding Author E-mail Address [ylliang@stanford.edu]
}




\begin{abstract}
Electrochemical impedance spectroscopy (EIS) is commonly used to investigate the kinetics of mixed ionic-electronic conductor (MIEC) electrodes. Across various applications, MIECs exhibit ionic (e.g., insertion) and/or electronic (e.g., electrocatalytic) charge transfer reactions at the electrode/electrolyte interface. Bulk storage and transport of charge carriers also couple with these interfacial reactions. Here, we build a generalized, physics-based impedance model for MIECs with an ion-blocking current collector and explore how bulk and interfacial resistance, chemical capacitance, and DC polarization affect the impedance response. Using transition metal hydroxides as a case study, we provide guidance on extracting reaction kinetics or bulk resistance from the Nyquist plots in interface- or bulk-controlled conditions, respectively. Generalizing our EIS analysis enables a robust analysis of MIEC kinetics for a diverse set of systems.
\end{abstract}

\title{
Introduction
}

Mixed ionic-electronic conductors (MIECs) are used as electrodes in many electrochemical energy-conversion and storage applications, including battery and pseudocapacitor electrodes, ${ }^{1-7}$ electrocatalysts in aqueous electrolytes, ${ }^{2,8-12}$ and solid-oxide fuel cell (SOFC) electrodes. ${ }^{13-16}$ The ionic and electronic charge carriers contribute to bulk conduction as well as chemical diffusion. Interfacial reactions in these electrodes are often selective, involving only the ionic or the electronic species. For example, ideal battery and pseudocapacitor electrodes source ions at the MIEC/electrolyte interface, compensating the charge with the electrons sourced from the current collector/MIEC interface. ${ }^{1,5,6}$ Some MIECs, such as electrocatalysts, may involve both ion and electron transfers at the MIEC/electrolyte interface due to the presence of redox couples in the electrolyte, such as $\mathrm{O}_{2(\mathrm{~g})} / \mathrm{OH}^{-} .16$

Electrochemical impedance spectroscopy (EIS) is used extensively to probe kinetics of MIEC electrodes. ${ }^{4,17-26}$ As a powerful alternating current (AC), frequency-domain method, EIS assesses electrode kinetics at open-circuit voltage and under an applied bias. Fitting models to EIS data can yield bulk (chemical) kinetics, interfacial capacitance, and interfacial reaction 
resistances. Several physics-based EIS models have been developed for MIEC electrodes in various modes of operation (i.e., different boundary conditions), including chemical storage and gas (e.g., $\mathrm{O}_{2}$ ) insertion in SOFC electrodes. ${ }^{17-19,23,25-29}$ The fundamentals have been extended to battery applications ${ }^{23,30-35}$ and pseudocapacitors. ${ }^{4,24}$ However, previous models have yet to consider simultaneous electronic and ionic interfacial reactions coupled with bulk transport across orders of magnitudes, limiting their generalizability in analyzing kinetic parameters in various systems.

In this study, we build on the bulk transport and chemical storage models derived in previous works and develop a one-dimensional EIS model for MIEC electrodes with two interfaces. At the MIEC/electrolyte interface, both ions and electrons can cross the boundary (controlled by their respective rate constants); at the current collector/MIEC interface, only electrons can transfer. This general model describes electrochemically-driven chemical storage (e.g., in battery electrodes), electron transfer reactions (e.g., in electrocatalysts), and mixed cases. These boundary conditions are similar to those of a Hebb-Wagner polarization cell. ${ }^{36-38}$ To visualize the model, we map these boundary conditions to equivalent circuits. The advantage of a physics-derived equivalent circuit is that one can easily manipulate material parameters and observe the changes in the calculated impedance. Using our model, we calculate the EIS impedance across a wide range of material parameters and draw correlations between the material properties and the features in calculated Nyquist plots. Understanding how these parameters affect the Nyquist plot provides insight into the EIS data without using phenomenological circuit elements, such as the constant phase element (CPE). ${ }^{18,19}$

Herein, we consider a specific MIEC material system that encompasses all features of interest mentioned above: first-row transition metal (TM) hydroxides in alkaline aqueous electrolytes. TM hydroxides, most notably nickel, cobalt, and iron hydroxides and their combinations, have been widely employed in energy storage (e.g., nickel-metal hydride batteries) and conversion (e.g., oxygen evolution electrocatalysts). ${ }^{12,39-43} \mathrm{TM}$ hydroxides are highly active electrocatalysts for the oxygen evolution reaction (OER) at the MIEC/alkaline electrolyte interface. ${ }^{12,39,40,42,43}$ Additionally, TM hydroxides function as battery electrodes in alkaline electrolytes, utilizing redox-coupled proton insertion/de-insertion. The hydroxide ions in the electrolyte react with the protons sourced from the bulk of the TM hydroxide at the 
MIEC/electrolyte interface. ${ }^{12,40,41,44,45}$ Additional complexity arises from proton insertion/deinsertion, which occurs roughly at the same potential as the onset of OER (between 1.3 and $1.5 \mathrm{~V}$ vs. RHE). Even though the migration of both protons and electronic species in the bulk has long been recognized, ${ }^{46-49}$ few impedance analyses have categorized this class of materials as a MIEC. In one such study, Mancier et al. built a MIEC model for nickel hydroxide to study its bulk redox process, yet the OER at the MIEC/electrolyte interface was not considered. ${ }^{46}$ Here we model TM hydroxides as MIECs, considering the migration of both the ionic and electronic carriers in the bulk as well as proton insertion/de-insertion and the OER occurring concurrently at the MIEC/electrolyte interface.

\section{Model}

We start by establishing a reaction-transport model of a one-dimensional slab of a MIEC electrode. As shown in Figure 1a, this electrode slab, with a thickness of $L$, is placed between a purely electron-conducting (ion-blocking) current collector (at position $x=0$ ) and a purely ionconducting (electron-blocking) electrolyte (at $x=L$ ). To consider both chemical storage (battery electrode) and electron transfer reactions (electrocatalyst), we allow both electronic and ionic charge carriers to undergo their respective interfacial reactions at the MIEC/electrolyte interface. The ability to transfer electrons between the MIEC and the electrolyte implies that the electrolyte contains an electron donor/acceptor redox couple. For simplicity, we neglect mass transport limitation within the electrolyte, and approximate the electrolyte impedance as a pure resistance. The impedance effects of electrolyte mass transport can be found in previous textbook and literature examples and can be added to the model in a straightforward manner. ${ }^{50-52}$

Although the model developed in this study can be generalized to describe any MIEC electrode, we adopt the specific example of TM hydroxide in alkaline electrolytes for easier understanding. As mentioned above, the bulk electronic and ionic carriers are electron-holes and proton vacancies, ${ }^{53-55}$ respectively. With the hydroxide ions in the electrolyte, the ionic charge transfer reaction exchanges protons across the MIEC/electrolyte interface. The electron exchange reaction is coupled with the $\mathrm{O}_{2(\mathrm{~g} /} / \mathrm{OH}^{-}$conversion in the electrolyte. Figure $1 \mathrm{~b}$ depicts these two reactions serving as the boundary condition at the MIEC/electrolyte interface. 


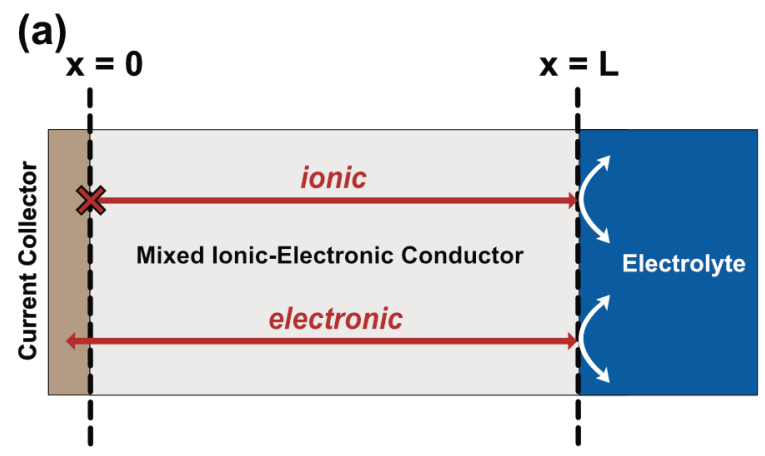

(b)

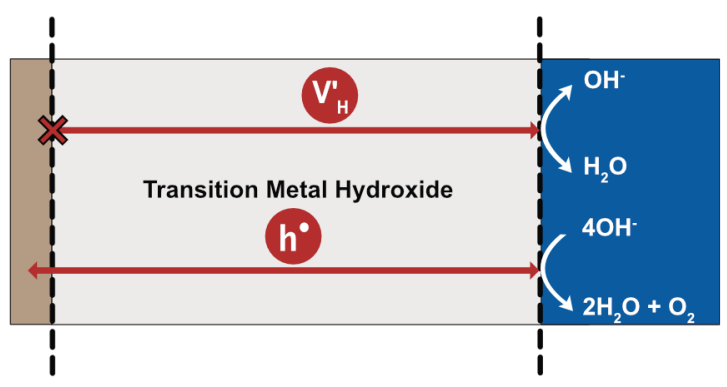

Figure 1. (a) Diagram illustrating MIEC on an ion-blocking current collector. (b) Specific charge carriers and reactions for transition metal hydroxide model in this study, with holes as the electronic carrier and proton vacancies as the ionic carrier.

\section{Charge transfer at Current Collector/MIEC Interface}

As depicted in Figure 1, the MIEC/current collector interface enables the selective transfer of electrons from the circuit while blocking ions. We can write a pseudo-chemical reaction to represent this electron transfer process:

$$
e^{-}(C C)+h^{\bullet} \rightarrow \text { null }
$$

Here, the subscript " $c c$ " denotes current collector, and $h^{\bullet}$ denotes the localized holes in the MIEC (TM hydroxide), written in the Kröger-Vink notation. We treat localized electron-holes in the MIEC as a dilute and ideal solid solution. For simplicity, we assume that the interfacial electron-transfer impedance at the current collector/MIEC interface is negligible. 


\section{Reactions at MIEC/Electrolyte Interface}

TM hydroxides exhibit OER activity at the MIEC/electrolyte interface under anodic conditions. The OER in an alkaline solution is given as:

$$
O H_{(a q)}^{-}+h^{\bullet} \rightarrow \frac{1}{4} O_{2(g)}+\frac{1}{2} H_{2} O_{(l)}
$$

The reaction free energy can then be expressed in terms of component electrochemical $\left(\widetilde{\mu}_{i}\right)$ and chemical $\left(\mu_{i}\right)$ potentials:

$$
\Delta \mu_{O E R, r x n}=\frac{1}{4} \mu_{O_{2(g)}}+\frac{1}{2} \mu_{H_{2} O_{(l)}}-\widetilde{\mu}_{h^{\bullet}}-\widetilde{\mu}_{O H_{(a q)}^{-}}
$$

The electrochemical potential can be written in a reduced form by dividing by its formal charge, $z_{i} e$, where $z_{i}$ is the charge number and $e$ is the elementary charge. The reduced form, $\widetilde{\mu}_{i}^{*}=\frac{\widetilde{\mu}_{i}}{z_{i} e}$, takes the same units as the electric potential. We can divide Equation 3 by $z_{i} e$ and rewrite the reaction potential as a product of current and resistance (assuming linear behavior over small voltage perturbation):

$$
J_{O E R}^{\text {charge }} R_{O E R}^{\perp}=-\frac{1}{4 e} \mu_{O_{2(g)}}-\frac{1}{2 e} \mu_{H_{2} O_{(l)}}+\widetilde{\mu}_{h^{\bullet}}^{*}-\widetilde{\mu}_{O H_{(a q)}^{-}}^{*}
$$

where $J_{O E R}^{\text {charge }}$ is the areal current density, and $R_{O E R}^{\perp}$ denotes the charge-transfer resistance of the OER, which we consider as a material constant (i.e., resistive behavior). In reality, $R_{O E R}^{\perp}$ depends on the MIEC/electrolyte interfacial composition and applied voltage and can be nonlinear over a wide range of applied voltage.

In addition to the OER, TM hydroxides also interact with the electrolyte by exchanging protons. We treat proton vacancies in the MIEC the same way we have treated electrons: as a dilute and ideal solid solution. Using the Kröger-Vink notation, the proton exchange reaction is written as:

$$
H_{H}^{\times}+O H_{(a q)}^{-} \rightleftharpoons H_{2} O_{(l)}+V_{H}^{\prime}
$$

where $H_{H}^{\times}$and $V_{H}^{\prime}$ denote hydrogen atom and proton vacancies, respectively. Analogous to Equation 4, we can write the following boundary condition for the proton exchange reaction: 


$$
J_{\text {proton }}^{\text {charge }} R_{\text {proton }}^{\perp}=-\frac{1}{e} \mu_{H_{2} O_{(l)}}+\widetilde{\mu}_{V_{H}^{\prime}}^{*}-\widetilde{\mu}_{O H_{(a q)}^{-}}^{*}
$$

where $J_{\text {proton }}^{\text {charge }}$ represents the areal current density from the proton exchange reaction, of which $R_{\text {proton }}^{\perp}$ is the ionic charge-transfer resistance. Unlike the OER reaction, the proton exchange reaction cannot reach steady state because of the ion-blocking boundary condition at the current collector/MIEC interface, so the proton flux must cease after some time (i.e., $J_{\text {proton }}^{\text {charge }}=0$ at $t \rightarrow$ $\infty)$

To maintain electroneutrality in the MIEC, the proton insertion/de-insertion must couple to charge compensation through a redox process in the MIEC (e.g., TM redox in TM hydroxide). The redox can be accomplished electrochemically (via electron-transfer at the current collector/MIEC interface) or chemically (via electron-transfer at the MIEC/electrolyte interface, where the proton exchange also occurs). The former case describes the ideal battery electrode, whereas the latter describes a so-called self-discharging battery.

In the case of the TM hydroxide, with DC polarization, ionic and electronic exchange reactions happen in parallel. Once the DC polarization is removed, the OER reaction and the proton exchange reaction (Equation 2 and 5) occur in tandem. The combination of the two reactions describes the protonating self-discharge process:

$$
\frac{1}{2} H_{2} O_{(l)}+h^{\bullet}+V_{H}^{\prime} \rightarrow \frac{1}{4} O_{2(g)}+H_{H}^{\times}
$$

This reaction satisfies electroneutrality in the MIEC. The corresponding boundary condition is given as:

$$
J_{\text {chem }}^{\text {charge }} R_{\text {chem }}^{\perp}=-\frac{1}{4 e} \mu_{O_{2(g)}}+\frac{1}{2 e} \mu_{H_{2} O_{(l)}}+\widetilde{\mu}_{h^{\bullet}}^{*}-\widetilde{\mu}_{V_{H}^{\prime}}^{*},
$$

We note that the resistance of the chemical proton insertion redox reaction $\left(R_{\text {chem }}^{\perp}\right)$ is the sum of the OER and proton exchange resistances (resistances in series):

$$
R_{\text {chem }}^{\perp}=R_{O E R}^{\perp}+R_{\text {proton }}^{\perp}
$$

Equation 7 is a chemical reaction where both holes and protons cross the MIEC/electrolyte interface with no net transfer of charge. In other words, proton insertion can 
occur without electrons passing through the external circuit via the current collector; the electronic charge is sourced from the $\mathrm{O}_{2} / \mathrm{OH}^{-}$redox couple in the electrolyte. Once the applied anodic polarization is removed, protons will insert and reduce the hydroxide, while oxygen gas is generated through a purely chemical path, returning the MIEC to a hydroxide form. The selfdischarging mechanism has been previously studied in detail in batteries with nickel hydroxidepositive electrodes, ${ }^{56-59}$ and one recent study utilized this reaction to generate oxygen chemically ${ }^{60}$ Such electrochemical ion insertion followed by a chemically-driven catalytic reaction can also be found in other MIEC catalytic systems. ${ }^{8,61-64}$

The boundary conditions examined in this work result in three distinct operating regimes for the MIEC. At steady state with an applied DC bias (static $V_{H}^{\prime}$ profile), the ion-blocking nature of the current collector means that only electron current can flow through the MIEC to facilitate electrocatalysis at the MIEC/electrolyte interface (e.g., OER). Away from steady-state (transient $V_{H}^{\prime}$ profile), ions are inserted into or removed from the MIEC, which is charge-compensated by a purely chemical process in the electrolyte (i.e., a self-discharging battery, Figure 2a, reactions in series) or by electron flow from the current collector (i.e., an ideal battery, Figure $2 b$, reactions in parallel). 55 
(a)

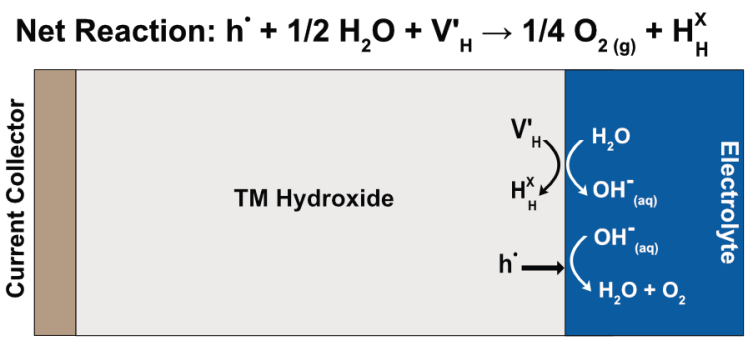

(b)

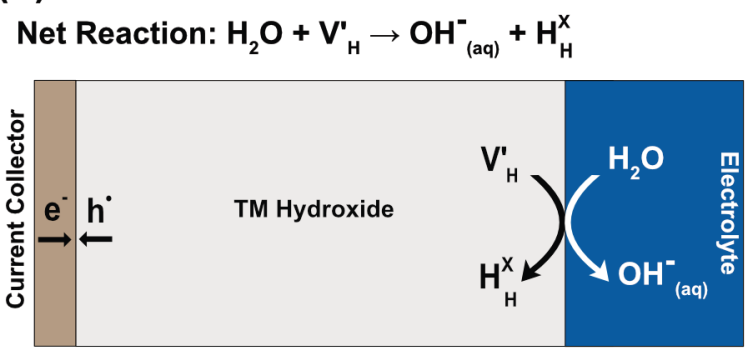

(c)

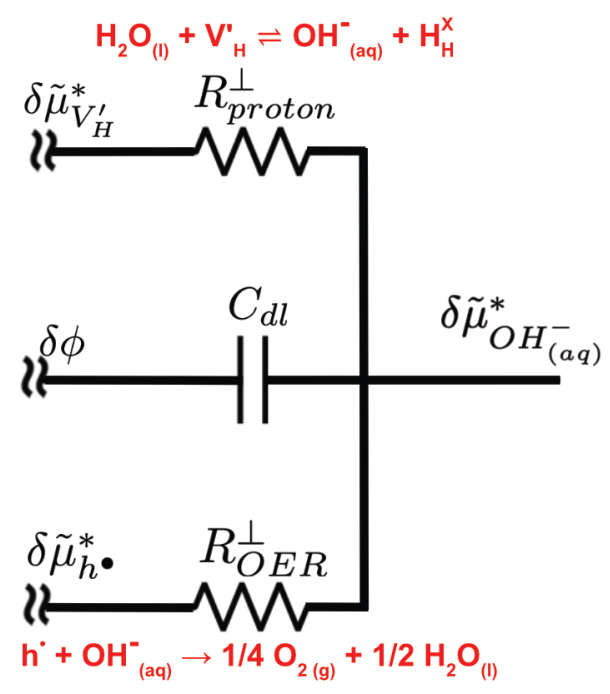

Figure 2. Illustration of the charge compensation of proton insertion through (a) chemically driven OER reaction; and (b) current flow from the current collector, an ideal battery operation. The color scheme is the same as Figure 1. (c) Equivalent circuit of the process at the MIEC/electrolyte interface, including proton exchange (top rail), oxygen evolution (bottom rail), and the double-layer (middle rail), under small AC perturbation. The left side of the circuit connects to the bulk MIEC, and the right side is the electrolyte. 


\section{Modeling the Bulk Properties}

Having defined the boundary conditions, we model bulk transport in the MIEC using the well-established Poisson-Nernst-Planck approach. ${ }^{25,26,51,65,66}$ For completeness, we review the key equations below. The generalized diffusion-drift equation for each charge carrier (i.e., ions, $V_{H}^{\prime}$, and electrons, $\left.h^{\bullet}\right)$ is written as ${ }^{18,51}$

$$
J_{i}^{\text {charge }}(x, t)=-z_{i} e D_{i} \frac{\partial c_{i}(x, t)}{\partial x}-\sigma_{i}(x, t) \frac{\partial \phi(x, t)}{\partial x}
$$

where $t$ is time and $\phi$ is the electric potential profile in the electrode. The parameter $D_{i}$ is the self-diffusion constant, $c_{i}$ is the charge carrier concentration, $\sigma_{i}$ is the partial electrical

conductivity, and $J_{i}^{\text {charge }}$ is the current density contributed by the carrier species $i$. With the Nernst-Einstein relation, the Equation 10 can also be written as:

$$
J_{i}^{\text {charge }}(x, t)=-z_{i} e D_{i} \frac{\partial c_{i}(x, t)}{\partial x}-\frac{\left(z_{i} e\right)^{2} D_{i} c_{i}(x, t)}{k_{B} T} \frac{\partial \phi(x, t)}{\partial x}
$$

The second equation is the Poisson equation which dictates the distribution of charged species within the MIEC:

$$
-\varepsilon_{r} \varepsilon_{0} \frac{\partial^{2} \phi(x, t)}{\partial x^{2}}=\sum_{i} z_{i} e c_{i}(x, t)
$$

where $\varepsilon_{r}$ is the dielectric constant and $\varepsilon_{0}$ is the vacuum permittivity. The final equation guarantees mass/charge conservation and the continuity of each migrating species within the bulk MIEC:

$$
z_{i} e \frac{\partial c_{i}(x, t)}{\partial t}+\frac{\partial}{\partial x} J_{i}^{\text {charge }}(x, t)=0
$$

We then sum up the contributions of all the carrier species in Equation 13 before inserting this sum into the Poisson equation. Then, integrating the resulted equation with respect to position $(x)$ gives the conservation of total charge flux $J_{\text {Total }}^{\text {charge }}$ :

$$
-\frac{\partial}{\partial t}\left[\varepsilon_{r} \varepsilon_{0} \frac{\partial \phi(x, t)}{\partial x}\right]+\sum_{i} J_{i}^{\text {charge }}(x, t)=J_{\text {Total }}^{\text {charge }}(t)
$$


The first term in this equation is the displacement flux, $J_{\text {dis }}$.

We apply the local electroneutrality approximation to the steady-state profile of the MIEC. ${ }^{67-69}$ The explicit derivation is included in the supplementary information (Equation A-1A-10). The approximation states that

$$
c_{h} \cdot(x)=c_{V_{H}^{\prime}}(x) \quad \forall x \in[0, L] \quad .
$$

\section{Steady State}

At steady state under DC polarization, the proton exchange reaction reaches equilibrium with no net proton inserted/de-inserted as the result of an ion-blocking electrode. The timescale a MIEC electrode requires to reach DC steady state depends on the chemical diffusivity. Thus, $J_{V_{H}^{\prime}}^{\text {charge }}=0$ and the OER reaction is the only reaction contributing to the measured charge flux $\left(J_{h} \cdot\right)$

We can re-write Equation 11 to find the relation between the concentration profile of holes and vacancies with the potential as

$$
\left\{\begin{array}{c}
\frac{\partial \phi}{\partial x}=\frac{k_{B} T}{e c_{V_{H}^{\prime}}} \frac{\partial c_{V_{H}^{\prime}}}{\partial x} \\
J_{h^{\bullet}}^{\text {charge }}=-e D_{h^{\bullet}} \cdot \frac{\partial c_{h^{\bullet}}}{\partial x}-\frac{e D_{h^{\bullet} c_{h^{\bullet}}}}{c_{V_{H}^{\prime}}} \frac{\partial c_{V_{H}^{\prime}}}{\partial x}
\end{array} .\right.
$$

Solving Equation 16 results in the steady-state concentration profiles for each charge carrier and the electrostatic potential profile:

$$
\left\{\begin{array}{c}
c_{h} \cdot(x)=c_{V_{H}^{\prime}}(x)=a\left(x-\frac{L}{2}\right)+b \\
\phi(x)=\frac{k_{B} T}{e}\left[\ln \left(\frac{b}{a}+\left(x-\frac{L}{2}\right)\right)\right]
\end{array}\right.
$$

Here, $a$ and $b$ are constants that depend on the boundary conditions (see supplementary information).

The total bulk resistance can either be calculated by integrating $1 / \sigma_{i}$ across $0 \leq x \leq L$ or measured through other means. For the rest of the main text, we will proceed with the total bulk ionic and electronic resistance, $R_{V_{H}^{\prime}}$ and $R_{h^{\bullet}}$, respectively. 


\section{Interfacial Equivalent Circuit}

Assuming that the perturbation in EIS is sufficiently small, we linearize the equations by taking only the zeroth-order term (steady-state solution in DC) and the first-order term (oscillation term) of the Taylor expansion in each equation. Looking at only the perturbation term in the boundary conditions and assuming fast mass transport in the bulk electrolyte results in:

$$
\begin{gathered}
\delta J_{O E R}(t) R_{O E R}^{\perp}=\delta \widetilde{\mu}_{h^{*}}^{*}(t)-\delta \widetilde{\mu}_{O H_{(a q)}^{-}}^{*}(t) \\
\delta J_{\text {proton }}(t) R_{\text {proton }}^{\perp}=\delta \widetilde{\mu}_{V_{H}^{\prime}}^{*}(t)-\delta \widetilde{\mu}_{O H_{(a q)}^{-}}^{*}(t)
\end{gathered}
$$

We also consider the chemically-driven proton insertion (i.e., self-discharging, Equation 7) by combining Equation 18 and 19:

$$
\delta J_{O E R}(t) R_{O E R}^{\perp}=\delta J_{\text {proton }}(t) R_{\text {proton }}^{\perp}+\delta \widetilde{\mu}_{h^{\bullet}}^{*}(t)-\delta \widetilde{\mu}_{V_{H}^{\prime}}^{*}(t)
$$

The double-layer capacitance of charged species at the MIEC/electrolyte interface must also be addressed, as it contributes to the interfacial impedance response. Given the fast replenishment of the electrolyte, we describe the double-layer capacitance $\left(C_{d l}\right)$ by a simplified Helmholtz plane model, which depends on the structure of the compact layer at the liquid interface (the space-charge region in the solid is not considered):

$$
C_{d l}=\frac{\varepsilon_{0} \varepsilon_{\text {electrolyte }}}{l_{c}}
$$

Here, $\varepsilon_{\text {electrolyte }}$ is the dielectric constant of the electrolyte and $l_{c}$ is the thickness of the compact layer. ${ }^{70}$ Under small perturbation, $C_{d l}$ is assumed to be constant in the linear regime.

To visualize the coupling between OER, proton exchange reaction, and double-layer capacitance, an equivalent circuit is shown in Figure 2c. The top and bottom horizontal rail represent the transport of proton vacancies and electron holes, respectively. The middle rail represents the displacement current density. Since the OER reaction involves only electron transfer (Equation 18), $R_{O E R}^{\perp}$ connects the hole-conducting rail to the electrolyte. Similarly, the 
proton exchange reaction (Equation 19) involves only the ion-conducting rail. As we approximate $C_{d l}$ as constant, it connects to the displacement current flux rail. ${ }^{18,65,66} \mathrm{On}$ the electrolyte side, both reactions involve only the hydroxide ion; therefore, $R_{O E R}^{\perp}$ and $R_{p r o t o n}^{\perp}$ are connected and share the same potential. Moreover, because the model assumes that the concentration of hydroxide ions in the electrolyte remains constant, the right side of $C_{d l}$ is also connected to the hydroxide conducting rail. Similar models in the literature have also utilized the same equivalent circuit to map the boundary condition. ${ }^{65,66}$

\section{Bulk Equivalent Circuit}

The linear approximation of the bulk transport equations has been derived in literature, ${ }^{25,26,65,66}$ and a simplified version is shown in the Supplementary Information Equation A11-A13. The total chemical capacitance $\left(C_{c h e m}\right)$ reflects the change in stoichiometry with chemical potential. The dielectric capacitance $\left(C_{d i s}\right)$ reflects the polarizability of the MIEC. These components build up a variant of the transmission-line circuit. ${ }^{18,19}$ Together with the boundary equivalent circuit developed earlier (Figure 2c), Figure 3 shows the complete equivalent circuit for a TM hydroxide material system, considering both the OER and proton exchange reaction at the MIEC/electrolyte interface.

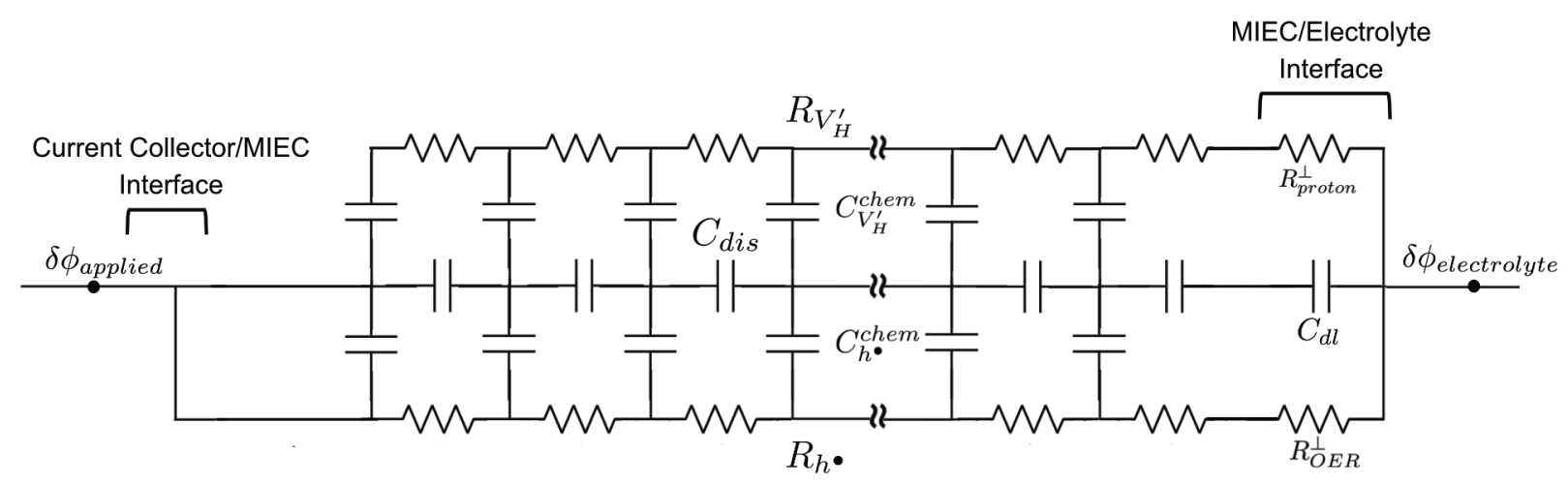

Figure 3. Full equivalent circuit of the MIEC. The current collector is located to the left of the circuit, and the electrolyte is located to the right.

\section{Non-dimensionalization \& Regimes}

We simplify the equivalent circuit by considering three sets of non-dimensional materials properties. First, we consider the boundary condition at the MIEC/electrolyte interface; 
specifically, the ratio of electronic to ionic resistance $\left(R_{O E R}^{\perp} / R_{\text {proton }}^{\perp}\right)$. Second, we consider the mechanism of energy storage, bulk vs. interfacial $\left(C_{c h e m} / C_{d l}\right)$. Finally, we consider whether transport is bulk-controlled or interface-controlled $\left(R_{V_{H}^{\prime}} / R_{\text {proton }}^{\perp}\right.$ for ionic and $R_{h} \cdot / R_{O E R}^{\perp}$ for electronic species).

For the remainder of the paper, we simulate impedance across multiple materials regimes by varying each of the three non-dimensional quantities listed above. We note that an ideal battery electrode stores energy chemically in the bulk $\left(C_{c h e m} \gg C_{d l}\right)$ and blocks electrons at the MIEC/electrolyte interface $\left(R_{O E R}^{\perp} \gg R_{\text {proton }}^{\perp}\right)$. An ideal electrocatalyst, on the other hand, allows electrons to transfer efficiently at the MIEC/electrolyte interface to catalyze an electrochemical reaction $\left(R_{O E R}^{\perp} \ll R_{\text {proton }}^{\perp}\right)$.

\section{Numerical Calculations}

Numerical calculations were performed in MATLAB by discretizing the bulk into $N=$ 20000 components. ${ }^{25}$ Unless otherwise specified, the steady-state DC polarization was assumed to be 10 times the magnitude of the AC signal amplitude. The amplitude of the AC signal is set to a unitless value of $0.01 \mathrm{~V}^{*} e / k_{B} T$. The total dielectric capacitance is assumed to be $C_{d i s}=$ $C_{d l} / 10^{5}$. The simulated frequency range is chosen between $1 \mathrm{GHz}$ and $1 \mu \mathrm{Hz}$. Realizing that such range is practically infeasible, we also highlight a more realistic frequency range accessible in typical experiments $(1 \mathrm{MHz}$ to $1 \mathrm{mHz})$.

To compare the Nyquist plots across parameters spanning several orders of magnitude, we normalize the magnitude of the impedance arcs. As the frequency approaches zero, the impedance of all capacitor elements approaches infinity. As such, only the total electron-hole resistance and OER charge-transfer resistance contribute to the total DC impedance. Thus, we normalized the Nyquist plots by $R_{h} \cdot+R_{O E R}^{\perp}$. Note that this normalization fails when $R_{O E R}^{\perp}$ approaches infinity, such as in the case of an ideal battery electrode. ${ }^{17}$ 


\section{Results and Discussion}

\section{Interface-controlled MIEC}

To understand how ionic and electronic reactions at the MIEC/electrolyte interface contributes to the Nyquist plot, we first consider a MIEC rate limited by the interfacial reactions (i.e., bulk transport is fast). Under this assumption, the reduced equivalent circuit is shown in Figure 4a. We further consider two cases differentiated by the charge storage mechanism: the MIEC stores energy (1) in the bulk $\left(C_{\text {chem }} \gg C_{d l}\right)$ and (2) at the electrode/electrolyte interface

$\left(C_{c h e m} \ll C_{d l}\right)$. We simulate a variety of $C_{c h e m} / C_{d l}$ and $R_{O E R}^{\perp} / R_{\text {proton }}^{\perp}$ ratios. Case (1) represents an ion-insertion electrode and two arcs are observed (Figure 4b). Case (2) represents a noninsertion electrode and only one arc is observed on the Nyquist plot (Figure 4c-d).

In the bulk-storage case $\left(C_{c h e m} \gg C_{d l}\right)$, the higher frequency arc (out of the two arcs) has a capacitance $C_{d l}$ and a resistance that corresponds to $R_{O E R}^{\perp}$ and $R_{\text {proton }}^{\perp}$ connected in parallel:

$$
R_{M F}^{a r c}=\left(\frac{1}{R_{O E R}^{\perp}}+\frac{1}{R_{\text {proton }}^{\perp}}\right)^{-1}
$$

where the subscript MF stands for "mid frequency" (the notation of "high frequency" will appear in the next section). The feature can be seen for the blue curve in Figure $4 \mathrm{~b}$ (corresponding to $R_{O E R}^{\perp}=R_{\text {proton }}^{\perp}$ ), where the arc has a normalized resistance of 0.5 . On the other hand, the DC resistance (sum of the width of the two arcs) corresponds to $R_{O E R}^{\perp}$. It follows that the lower frequency arc has a resistance given by $R_{O E R}^{\perp}-R_{M F}^{a r c}$. In this frequency regime, the chemical capacitance dominates the impedance arc, though the circuit does not correspond to a standard parallel RC circuit. In an ideal battery electrode, $R_{O E R}^{\perp}$ can be very large; therefore, this lower frequency arc will appear as a blocking, purely capacitive feature. 
(a)

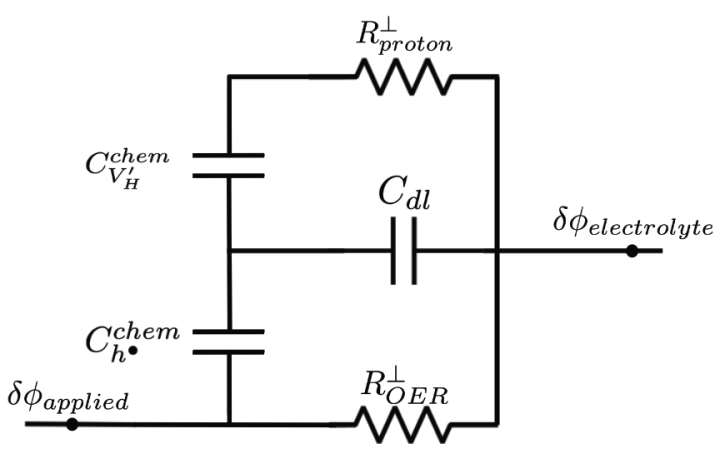

$\longrightarrow R_{\text {proton }}^{\perp} / R_{O E R}^{\perp}=10^{-2} \longrightarrow R_{\text {proton }}^{\perp} / R_{O E R}^{\perp}=1 \quad \longrightarrow R_{\text {proton }}^{\perp} / R_{O E R}^{\perp}=10^{2}$

(b) $C_{\text {chem }} / C_{d l}=10^{3}$

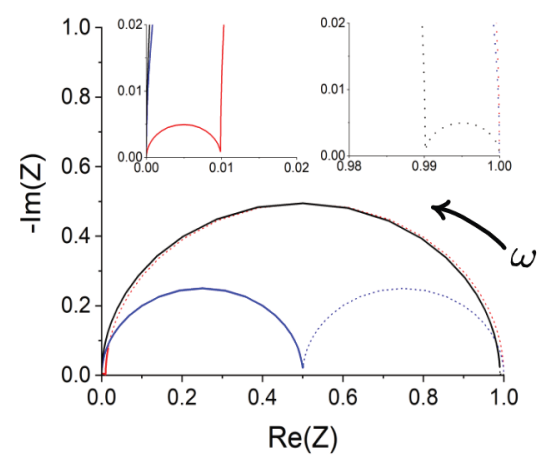

(c) $C_{\text {chem }} / C_{d l}=1$

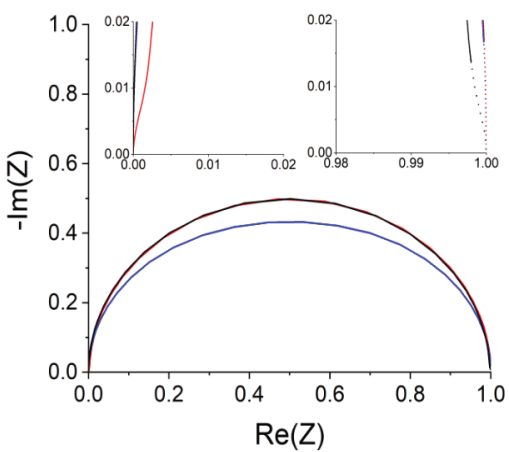

(d) $C_{\text {chem }} / C_{d l}=10^{-3}$

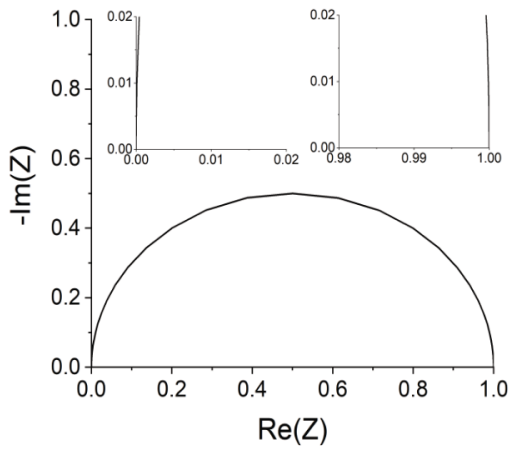

Figure 4. (a) Reduced equivalent circuit under the assumption of fast diffusion in the bulk material. (b)-(d) Nyquist plots of the calculated electrochemical impedance with different charge-transfer resistance ratios between proton exchange and OER. The simulated frequency range is chosen between $1 \mathrm{GHz}$ and $1 \mu \mathrm{Hz}$ (dotted lines). The result is normalized with the summation of resistance on the electronic rail (i.e., $R_{h}$. $+R_{O E R}^{\perp}$ ). Realizing that such range is practically infeasible, we also highlight a more realistic frequency range accessible in typical experiments (1MHz to $1 \mathrm{mHz})$, which is shown as solid lines. Each plot has two insets: the upper-left one zooms in on the high-frequency (low $\operatorname{Re}(Z)$ ) end, and the upper-right one zooms in on the low-frequency (high $\operatorname{Re}(Z)$ ) end. All three lines in (d) completely overlap with one another.

In the interfacial-storage case $\left(C_{c h e m} \ll C_{d l}\right)$, a single arc is consistently observed across many orders of magnitude for $R_{O E R}^{\perp}$ and $R_{\text {proton }}^{\perp}$ (Figure $4 \mathrm{~d}$ ), corresponding to a simple parallel $\mathrm{RC}$ circuit $\left(R_{O E R}^{\perp}\right.$ and $2 C_{\text {chem }}$ ). Interestingly, neither $C_{d l}$ nor $R_{\text {proton }}^{\perp}$ is reflected in the Nyquist plot. At first, this observation appears unintuitive given what is known for metallic electrodes since $R_{O E R}^{\perp}$ and $C_{d l}$ primarily describe the electrode/electrolyte interface. Because the value of $C_{d l}$ (relative to $C_{c h e m}$ ) is large, it is polarizable at a frequency in which the bulk stoichiometry is 
no longer responsive to either the electrical perturbation (i.e., the impedance of the chemical capacitors approaches infinity) or the flow of dielectric displacement current (i.e., the impedance of the bulk dielectric capacitors approaches infinity). In other words, for electrodes with substantial dielectric polarizability (i.e., non-metallic electrodes), charging and discharging of the double-layer requires either a change in carrier concentration or dielectric polarization in the bulk material. Similarly, protonation must also involve bulk composition change, so the arc width does not depend on $R_{\text {proton }}^{\perp}$

\section{Bulk-controlled MIEC}

When the bulk resistance in the MIEC electrode becomes non-negligible due to increased electrode thickness and/or decreased conductivity, carrier transport contributes to, or even dominates the Nyquist plots. Recall that our impedance simulations are performed under DC polarization. Just as in the case of Hebb-Wagner cells, the ion-blocking nature of the current collector interface/MIEC means that the steady-state ionic current is zero, and the bulk exhibits a potential drop due to the electronic current. As such, a bulk-controlled MIEC exhibits ionic and electronic concentration gradients according to Equation 17.

Figure 5 shows the Nyquist plots for a battery-electrode-like MIEC, where $R_{O E R}^{\perp} \gg R_{\text {proton }}^{\perp}$ and $C_{c h e m} \gg C_{d l}$. We vary the surface-to-bulk resistance ratios for both ions (columns) and electrons (rows). Unsurprisingly, with minimal bulk resistance for either charge carrier, Figure 5a reproduces the reaction-controlled case. As $R_{V_{H}^{\prime}}$ and/or $R_{h} \bullet$ increases, an additional high-frequency arc emerges. Using Figure $5 \mathrm{~b}$ as an example here, the width of the high-frequency arc, $R_{H F}^{a r c}$, is determined by the bulk electronic and ionic resistances connected in parallel:

$$
R_{H F}^{a r c}=\left(\frac{1}{R_{h^{\bullet}}}+\frac{1}{R_{V_{H}^{\prime}}}\right)^{-1}
$$

The mid-frequency arc represents the coupling between $C_{d l}$ and both interfacial reaction resistances, similar to the interfacial-controlled case. As a result, Equation 22 applies here and determines the width of this arc, $R_{M F}^{\operatorname{arc}}$. The low-frequency arc width, $R_{L F}^{\operatorname{arc}}$, dominated by the chemical capacitance, can then be expressed as 


$$
R_{L F}^{a r c}=\left(R_{O E R}^{\perp}+R_{h^{*}}\right)-\left(R_{H F}^{a r c}+R_{M F}^{a r c}\right) .
$$

where the first term is the DC resistance reflecting the ion-blocking nature of the current collector. When the OER resistance is large in a battery-electrode-like MIEC, $R_{L F}^{a r c}$ is enormous, making the arc largely inaccessible experimentally and appear as blocking.

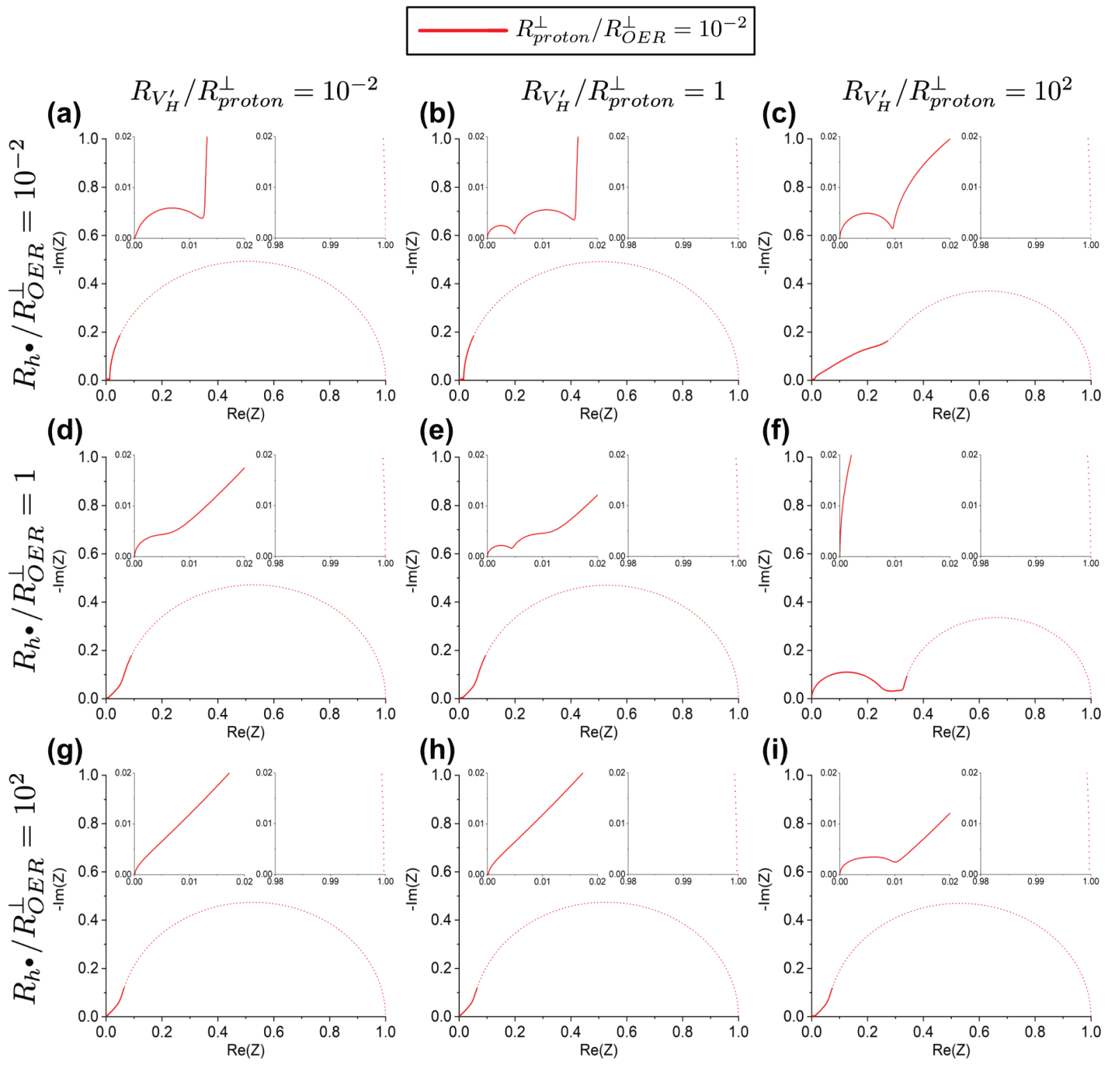

Figure 5. Calculated Nyquist plots with varying input parameters. $C_{c h e m} / C_{d l}$ is fixed at 1000 . The ratio between the proton vacancy bulk resistance to the charge transfer resistance of proton insertion/de-insertion, $R_{V_{H}^{\prime}} / R_{\text {proton }}^{\perp}$, changes from left to right; the ratio between hole bulk resistance to the OER resistance, $R_{h}$. $/ R_{O E R}^{\perp}$, varies from top to bottom. The ratio between two surface reactions is fixed at $R_{\text {proton }}^{\perp} / R_{O E R}^{\perp}=0.01$. Same presentation and normalization schemes as Figure 4. 
Under DC polarization, the mid-frequency arc corresponding to $R_{M F}^{a r c}$ is often not visible or overlaps substantially with the low-frequency arc. As can be seen in Figure $5 \mathrm{c}$ and other subplots with either large $R_{V_{H}^{\prime}}$ or $R_{h^{*}}$, the impedance arc due to the interfacial reaction might not be measurable or may need to be extrapolated. In these conditions, only the bulk resistance value can be confidently extracted from the Nyquist plot.

Another interesting observation while going down the columns in Figure 5 is the appearance of the so-called Warburg behavior in the low-frequency range. Warburg behavior models the diffusion-controlled electroactive species, and increasing the resistivity often leads to a more obvious Warburg feature on the Nyquist plot. ${ }^{70,71}$ As seen in Figure $5 \mathrm{~g}$, by increasing $R_{h}$. and coupling with large OER interfacial resistance, the impedance results in $45^{\circ}$ slope, a typical Warburg feature. Conversely, the appearance of the Warburg feature is not necessarily the case when going across the row in Figure 5 (e.g., increasing $R_{V_{H}^{\prime}}$ from $5 \mathrm{a}$ to $5 \mathrm{c}$ ). With the boundary condition of an ion-blocking current collector, the Warburg behavior is not necessarily present in the Nyquist when $R_{h}$ • is sufficiently small.

Figure 6 shows the diffusion-controlled Nyquist plots for $R_{O E R}^{\perp} \ll R_{\text {proton }}^{\perp}$, also in the scenario that $C_{\text {chem }} \gg C_{d l}$ (bulk-storage case). These boundary conditions are typical for electrocatalysts but can also describe non-ideal, self-discharging battery electrodes, such as the TM hydroxides in our case study. A substantial difference from Figure 5 is the absence of the Warburg element across all simulation conditions. Regardless of large $R_{V_{H}^{\prime}}$ and/or $R_{h^{*}}$, large bulk resistance does not give rise to a $45^{\circ}$ slope. with the ion-blocking nature at the current collector/MIEC interface, we speculate that this is because the sum of electronic bulk and interfacial resistance of the electronic species is smaller than that of ionic species (value listed in Table A-1). In this case, the majority of current flow favors the electronic rail and prevents the Warburg element from developing, though this claim requires further studies to confirm.

Across all bulk-controlled scenarios in which $C_{c h e m} \gg C_{d l}$, substantial bulk resistance causes substantial curve depression in the Nyquist plots (e.g., Figure $5 \mathrm{i}$ high-frequency inset and Figure 6i). This behavior is usually modeled with a CPE, representing the distribution of relaxation time constants due to inhomogeneity across the thickness of the electrode. ${ }^{51}$ Here, a large potential drop in the electrode results in an inhomogeneous distribution of chemical 
capacitance and resistance in the bulk. These differences contribute to CPE-like behavior, such as the high frequency arc in Figure 5i. As shown in Figure 7, removing the applied DC polarization restores the carrier concentration to homogenous distribution in the bulk material and eliminates the depression in the Nyquist plot. Complete results on the impedance response without DC bias are in the supplementary information (Figure A-2).

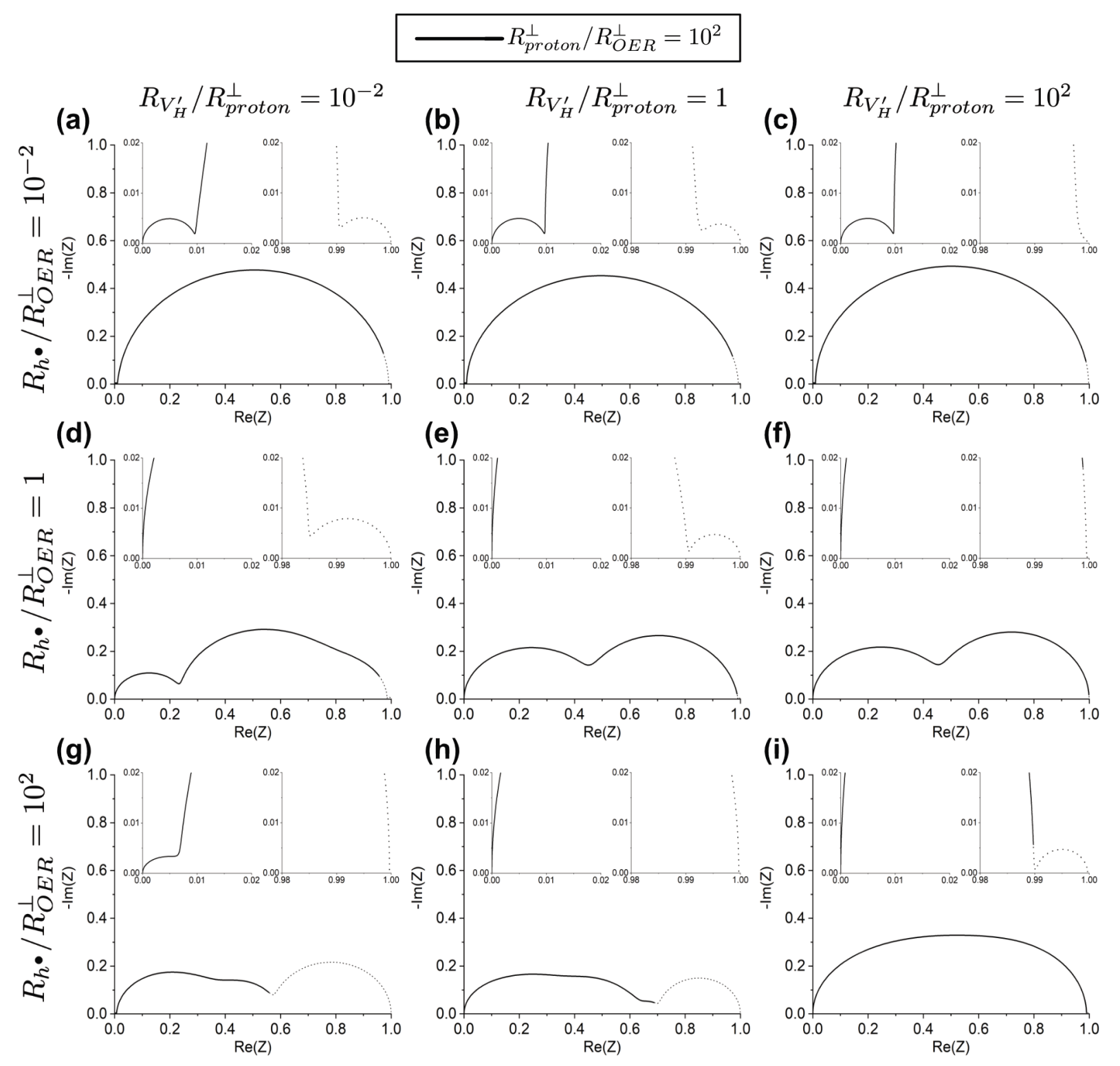

Figure 6. Calculated Nyquist plots with varying input parameters. Same inputs as Figure 5, except $R_{\text {proton }}^{\perp} / R_{O E R}^{\perp}=100$. Same presentation and normalization schemes as Figure 4. 
(a)

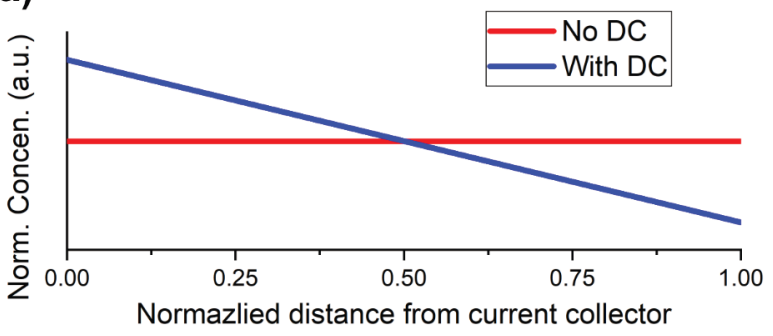

(b) With DC

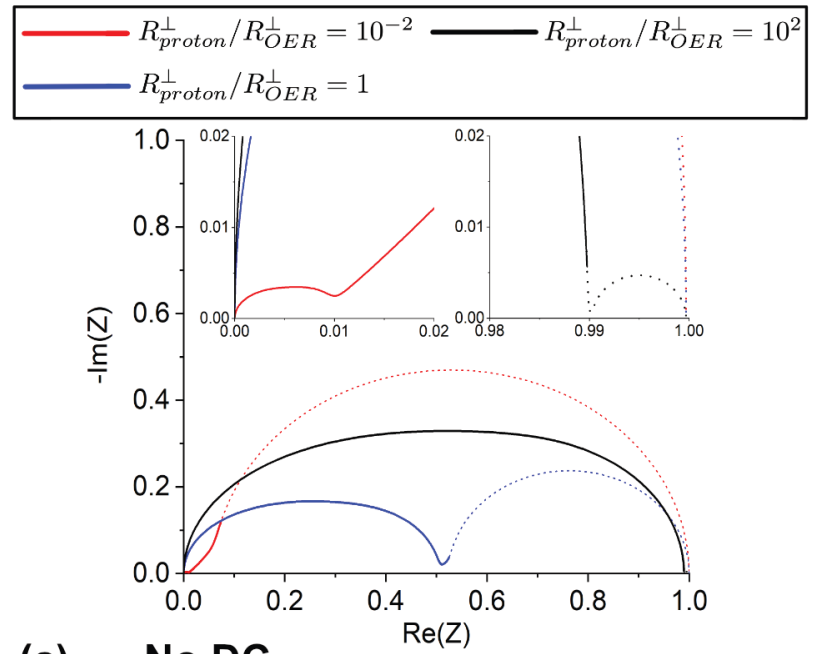

(c) No DC

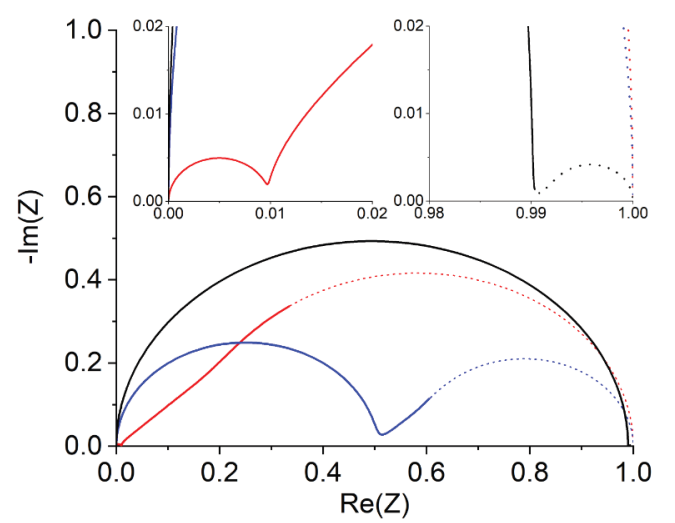

Figure 7. (a) The normalized concentration profile of charge carriers in the MIEC bulk with or without DC bias. The calculated Nyquist plot (b) with and (c) without applied DC. Parameters are set as $R_{h} \cdot R_{O E R}^{\perp}=100, R_{V_{H}^{\prime}} / R_{\text {proton }}^{\perp}=100$, and $C_{\text {chem }} / C_{d l}=1000$. Same presentation and normalization schemes as Figure 4. 
Besides the CPE-like features, other arc depressions and distortions are present when the bulk resistance is sufficiently large (Figure 5-6). For example, in Figure 5c, the mid-frequency arc (corresponding to $R_{M F}^{a r c}$ ) cannot be distinguished from the large, distorted bulk arc beyond $\operatorname{Re}(Z)=0.01$. These distortions and arc depressions are signs that (1) bulk resistance is dominating, and (2) the DC polarization is causing substantial inhomogeneity, though the exact mechanism is beyond this study's scope.
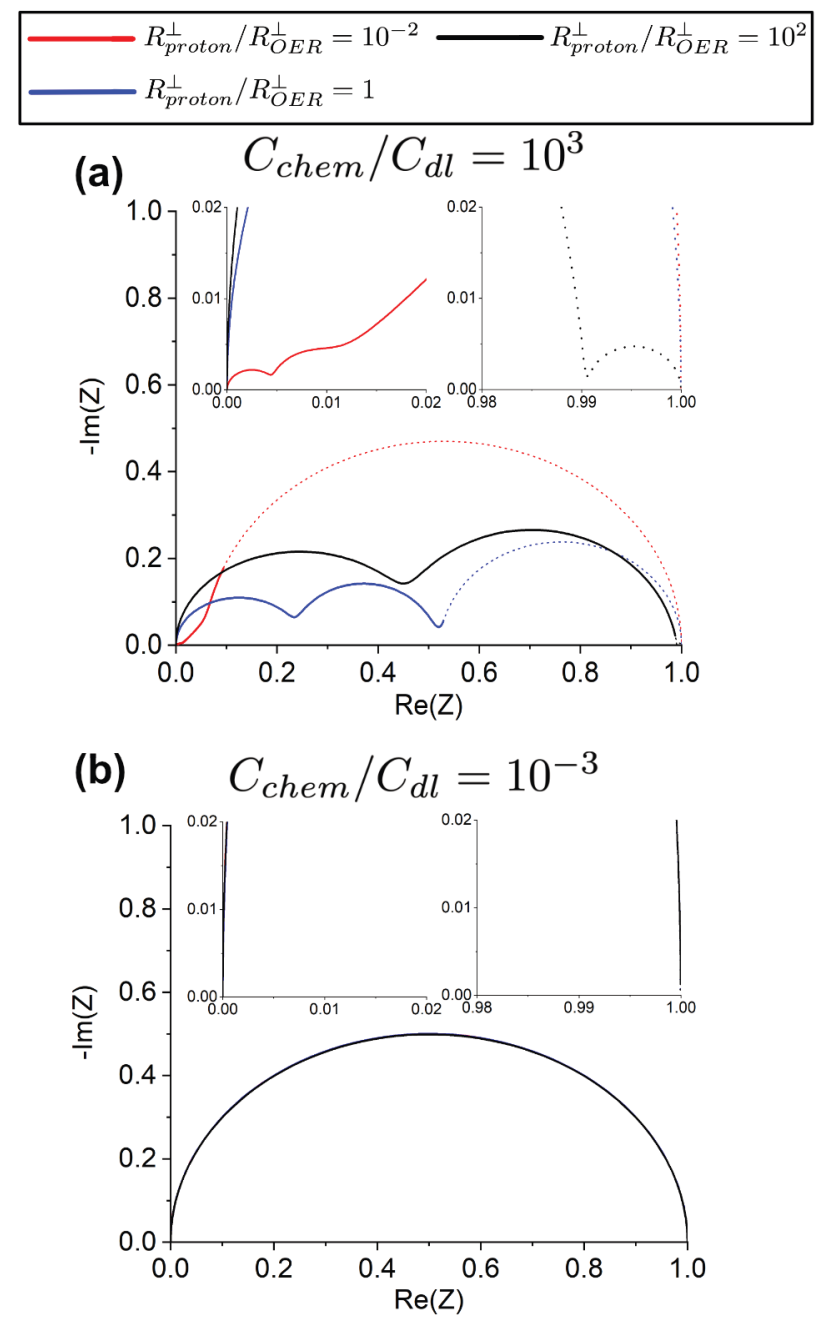

Figure 8. Calculated Nyquist plot with various $C_{\text {chem }} / C_{d l}$ ratios, $R_{h \cdot} / R_{O E R}^{\perp}=1, R_{V_{H}^{\prime}} / R_{\text {proton }}^{\perp}=$ 1 , and DC bias applied. 
So far, we have focused the discussion of bulk-controlled scenarios on MIECs with large chemical capacitance. When $C_{c h e m} \ll C_{d l}$, only one arc appears in the Nyquist plot, like the interface-controlled scenario (Figure 8). As explained previously, the EIS does not perturb the double-layer capacitance with a small chemical capacitance since neither the bulk carrier concentration nor dielectric polarization is responsive. Only $C_{c h e m}$ and the sum of $R_{O E R}^{\perp}$ and $R_{h}$. can be extracted from the Nyquist plot. The complete simulation results for $C_{c h e m} \ll C_{d l}$ can be found in Figure A-3.

\section{A Guide for Interpreting MIEC Nyquist Plots}

Even though this study focuses mainly on the impedance analysis of TM hydroxide electrodes, we emphasize the broad generalizability of the MIEC model. The model simulates various types of material behaviors, including ionic insertion (battery electrodes), electronic charge transfer (non-intercalating electrocatalysts), and materials with both properties (such as the TM hydroxide in this study). However, there is not a one-to-one correspondence between the Nyquist plots and the material properties, which makes it challenging to identify all desired parameters. Nonetheless, Figure 9 provides a general guideline for extracting resistance values

from the impedance measurements. This decision tree applies when $C_{c h e m} \gg C_{d l}$. When the double layer capacitance is large, the impedance measurement always results in a single arc on the Nyquist plot. 


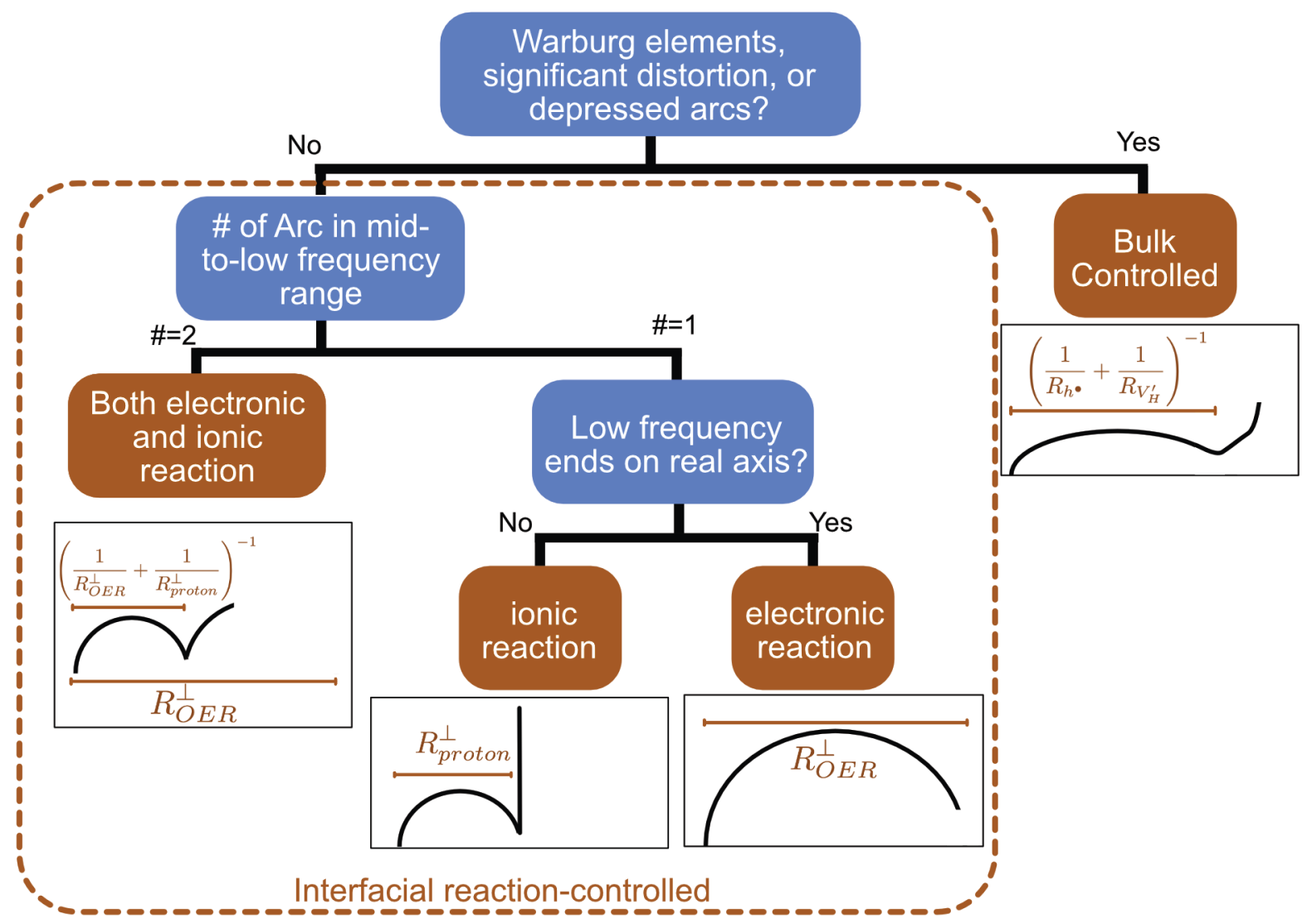

Figure 9. A decision tree on how one analyzes the resistance value based on the Nyquist plot features, assuming $C_{c h e m} \gg C_{d l}$.

When one observes a depressed semicircle and/or a Warburg-like feature, the MIEC electrode is bulk-controlled. As such, bulk transport properties are straightforwardly extracted from the high-frequency arc. On the other hand, if no distortion or Warburg-like feature is visible, the electrode is interface-controlled. If only one arc appears in the Nyquist plot, the interfacial reaction is dominated by just one of the charge-transfer reactions. For an ideal battery electrode $\left(R_{O E R}^{\perp} \gg R_{\text {proton }}^{\perp}\right)$, a single arc in the mid-frequency range is expected and the lowfrequency response is blocking. For an electrocatalyst $\left(R_{O E R}^{\perp} \ll R_{\text {proton }}^{\perp}\right)$, a single, semi-circle that ends on the real axis at low-frequency region is expected. Lastly, if the Nyquist plot shows two semi-circles, the MIEC/electrolyte interface exhibits comparable ionic and electronic resistances. 


\section{Conclusions}

In summary, we developed a general MIEC impedance model that considers concurrent ion-inserting and electrocatalytic reactions. Using TM hydroxides on an ion-blocking current collector as a case study, we coupled the bulk transport of ionic and electronic species to their respective ion exchange and OER reactions at the MIEC/electrolyte interface. Results from numerical calculation revealed how ionic and electronic reactions contributed to the impedance. Using these insights, we built a decision tree to guide the interpretation of impedance of MIEC electrodes. Finally, we highlight this MIEC model's versatility, encouraging further studies to adapt the fundamentals presented here to a broader range of systems.

\section{Acknowledgments}

The authors gratefully acknowledge financial support received through the Department of Energy, Office of Basic Energy Sciences, Division of Materials Sciences and Engineering under contract no. DE-AC02-76SF00515. We would also like to acknowledge that part of the computing for this project was performed on the Stanford Sherlock Cluster, maintained and supported by the Stanford Research Computing Center. The authors also thank Dr. Chia-Chin Chen for helpful discussions.

\section{Appendix}

\section{Solving Steady-State Concentration Profile for Charge Carriers}

Given that $J_{V_{H}^{\prime}}^{\text {charge }}(x)=0$ in our steady-state model, we can use the generalized transport equation (Equation 11 in main article) to solve for the distribution of ionic species in the electrode:

$$
\frac{\partial \phi}{\partial x}=-\frac{k_{B} T}{z_{V_{H}^{\prime} e c_{V_{H}^{\prime}}(x)}} \frac{\partial c_{V_{H}^{\prime}}(x)}{\partial x} .
$$


By plugging the equation back into the transport equation, $J_{h^{\bullet}}^{\text {charge }}$ can then be expressed

as

$$
J_{h^{\bullet}}^{\text {charge }}(x)=-z_{h^{\bullet}} e D_{h} \cdot \frac{\partial c_{h^{\bullet}}(x)}{\partial x}+\frac{z_{h^{\bullet}}{ }^{2} e D_{h} c_{h} \cdot(x)}{z_{V_{H}^{\prime}} c_{V_{H}^{\prime}}(x)} \frac{\partial c_{V_{H}^{\prime}}(x)}{\partial x} .
$$

With the conservation of mass (and thus charge) from Equation 13, under the steady-state condition, $\frac{\partial}{\partial x} J_{h^{\bullet}}^{\text {charge }}(x)=0$.

Given these boundary conditions, solving Equation 11-12 analytically is not straightforward. Local charge neutrality approximation is adopted here. Violation of the Poisson equation (Equation 12) is unavoidable when this method is applied. ${ }^{68}$ However, previous literature has adopted this approximation method to solve the concentration profile in a MIEC to a reasonable degree, within some acceptable error range. ${ }^{67,69}$

As stated in the name of this assumption, the net charge at every location within the bulk is zero. That is, $z_{h} \cdot c_{h} \cdot(x)+z_{V_{H}^{\prime}} c_{V_{H}^{\prime}}(x)=0$. Then, one can express Equation A-2 as

$$
J_{h^{\bullet}}^{\text {charge }}=\left(-z_{h^{\bullet}}+\frac{z_{h^{*}}}{z_{V_{H}^{\prime}}}\right) e D_{h^{\bullet}} \frac{\partial c_{h^{\bullet}(x)}}{\partial x} .
$$

Given that $J_{h^{\bullet}}^{\text {charge }}$ must be identical for all positions, $\frac{\partial c_{h^{\bullet}}}{\partial x}$ (and that of the proton vacancies) must be a constant value. That is,

$$
\left\{\begin{array}{c}
c_{h^{\circ}} \cdot(x)=a\left(x-\frac{L}{2}\right)+b \\
c_{V_{H}^{\prime}}(x)=-\frac{z_{h^{\bullet}}}{z_{V_{H}^{\prime}}}\left(a\left(x-\frac{L}{2}\right)+b\right)
\end{array}\right.
$$

Here, the scalar coefficients $a, b$ can be obtained through other boundary conditions (the total potential drop across the bulk and the bulk resistance of electronic species). With this linear concentration profile, Equation A-1 yield a potential profile: 


$$
\phi=\frac{-k_{B} T}{z_{V_{H}^{\prime}} e}\left[\ln \left(\frac{b}{a}+\left(x-\frac{L}{2}\right)\right)\right]
$$

and potential drop across the bulk material is given by:

$$
\Delta \phi=\frac{-k_{B} T}{z_{V_{H}^{\prime}} e}\left[\ln \left(\frac{b}{a}+\frac{L}{2}\right)-\ln \left(\frac{b}{a}-\frac{L}{2}\right)\right]=\frac{-k_{B} T}{z_{V_{H}^{\prime}} e} \ln \left(\frac{1+\frac{a L}{2 b}}{1-\frac{a L}{2 b}}\right) .
$$

Because of local charge neutrality, the Poisson equation states that the second derivative of the potential should be zero. However, Equation A-5 indicates that the left-hand side of Equation 12 is

$$
\frac{\partial^{2} \phi}{\partial x^{2}}=\frac{k_{B} T}{z_{V_{H}^{\prime}} e\left(x-\frac{L}{2}+\frac{b}{a}\right)^{2}} \neq 0
$$

The local charge neutrality demands that the right-hand side to be zero. Thus, the Poisson equation does not hold true.

Given the knowledge of total electronic resistance, $R_{h} \cdot$, we can use the Nernst-Einstein relation, which relates the conductivity $\left(\sigma_{i}\right)$ to the charge carrier concentration and its diffusion constant $\left(D_{i}\right)$, to derive the scalar constants in Equation A-4. The Nernst-Einstein relation states that: 25,51

$$
\sigma_{i}(x)=\frac{\left(z_{i} e\right)^{2} D_{i}}{k_{B} T} c_{i}(x)
$$

Then, integrating the inverse of conductivity yields the total area specific electronic resistance:

$$
R_{h^{\bullet}}=\int_{0}^{L} \frac{d x}{\sigma_{h^{\bullet}(x)}}=\frac{k_{B} T}{\left(z_{\left.h^{\bullet} e\right)^{2} D_{h^{\bullet}}}\right.} \frac{1}{a} \ln \left(\frac{1+\frac{a L}{2 b}}{1-\frac{a L}{2 b}}\right) .
$$

Subsequently, solving Equation A-7 and A-9 together yields the scalar value of a and b.

If one wishes to calculate $R_{i}(x)=1 / \sigma_{i}(x)$, which is the input for the impedance calculation of this study, using the electronic species as an example, one can write: 


$$
R_{h} \cdot(x)=R_{h} \cdot * \frac{1}{\ln \left(\frac{1+\frac{a L}{2 b}}{1-\frac{a L}{2 b}}\right)} * \frac{1}{\left(x-\frac{L}{2}\right)+\frac{b}{a}}
$$

Equation A-7 can yield the ratio between $a$ and $b$. In other words, Equation A-10 states a simple method to obtain the input parameters for solving the impedance model without explicitly knowing the diffusion coefficient of each charge carrier.

\section{Linear Approximation of The Bulk Properties}

The linear approximation of bulk properties has been previously derived in several works. $^{25,26,65,66}$ Here, only the related, simplified results are shown to connect to our boundary conditions. Full circuit development this work has referred to is in Lai's work. ${ }^{25}$ Under small AC perturbation, the equations governing charge transport and distribution (Equation 11-13,18) can be linearized for their first-order perturbation terms. Because the response to frequency modulation is of interest, Laplace transformation is performed to express these equations in the frequency domain:

$$
\begin{aligned}
& \delta J_{i}^{\text {charge }}(x, \omega)=-\sigma_{i}(x) \frac{\partial \delta \widetilde{\mu}_{i}^{*}(x, \omega)}{\partial x}+\frac{z_{i} e J_{i}^{\text {charge }}}{k_{B} T} \delta \mu_{i}^{*}(x, \omega) \\
& \frac{\partial \delta J_{i}^{\text {charge }}(x, \omega)}{\partial x}=-j \omega \frac{\left(z_{i} e\right)^{2} c_{i}(x)}{k_{B} T} \delta \mu_{i}^{*}(x, \omega) \\
& \delta J_{\text {dis }}(x, \omega)=-j \omega \varepsilon_{r} \varepsilon_{0} \frac{\partial \delta \phi(x, \omega)}{\partial x} \\
& \delta J_{\text {Total }}^{\text {charge }}(\omega)=\delta J_{\text {dis }}(x, \omega)+\sum_{i} \delta J_{i}^{\text {charge }}(x, \omega),
\end{aligned}
$$

where $j=\sqrt{-1}$ and $\omega$ is the angular frequency of the applied AC signal. Given that the local charge neutrality approximation may induce substantial error when the potential drop is sufficiently large, the steady-state DC is assumed to be small. The resulting electronic current density, $J_{h^{\bullet}}^{\text {charge }}$ is then neglected here. $J_{V_{H}^{\prime}}^{\text {charge }}$ is already zero with the steady-state condition.

Mapping of the AC response of bulk phenomenon (Equation A-11) to an equivalent circuit has appeared multiple times in the literature, as mentioned earlier. Building a transmission line model by interpreting each parameter in the equations as a circuit element results in: 


$$
\begin{aligned}
& \delta \widetilde{\mu}_{i}^{*}(x, \omega)-\delta \phi(x, \omega)=\delta \mu_{i}^{*}(x, \omega) \\
& R_{i}(x)=\frac{1}{\sigma_{i}(x)} \\
& Z_{i}^{\text {chem }}(x, \omega)=\frac{1}{j \omega C_{i}^{\text {chem }}(x)} \quad \text { where } \quad C_{i}^{\text {chem }}(x)=\frac{\left(z_{i} e\right)^{2} c_{i}(x)}{k_{B} T} \\
& Z_{d i s}=\frac{1}{j \omega C_{d i s}} \quad \text { where } \quad C_{d i s}=\varepsilon_{r} \varepsilon_{0} .
\end{aligned}
$$

Here, $R_{i}(x)$ represents the resistance imposed by that charge carrier, and $C_{i}^{\text {chem }}(x)$ is the chemical capacitance of the charge carrier at location $x$ and, as suggested by the concentration term, subject to the stoichiometry of the mobile ionic or electronic species rather than the electrostatic field. ${ }^{18}$ The total chemical capacitance of the bulk, $C_{c h e m}$, is the summation of all components in the bulk. Because the net charge of the bulk material must be neutral, the total chemical capacitance is identical for both the hole and the vacancy. One last variable in Equation A-12 is $C_{d i s}$, the dielectric capacitance of the material.

Solving the system of differential equations consisting of Equation A-12 with boundary conditions, Equation 18-19 from the main body, proves to be difficult. Instead, a numerical solution can be calculated by approximating the derivative terms in Equation A-11 with

$$
\frac{\partial y}{\partial x} \approx \frac{y_{n+1}-y_{n}}{x_{n+1}-x_{n}}=\frac{y_{n+1}-y_{n}}{l}, \text { where } l=x_{n+1}-x_{n}
$$

Here, we divide the material into $N$ discrete components and label each section with $n \in[1, N]$. Effectively, this turns the question into solving a system of linear equations, which can be handled easily with computers. Moreover, the discretized parameters in Equation A-11 can be described with circuit elements, enabling subsequent mapping of our TM hydroxide model to an equivalent circuit. 


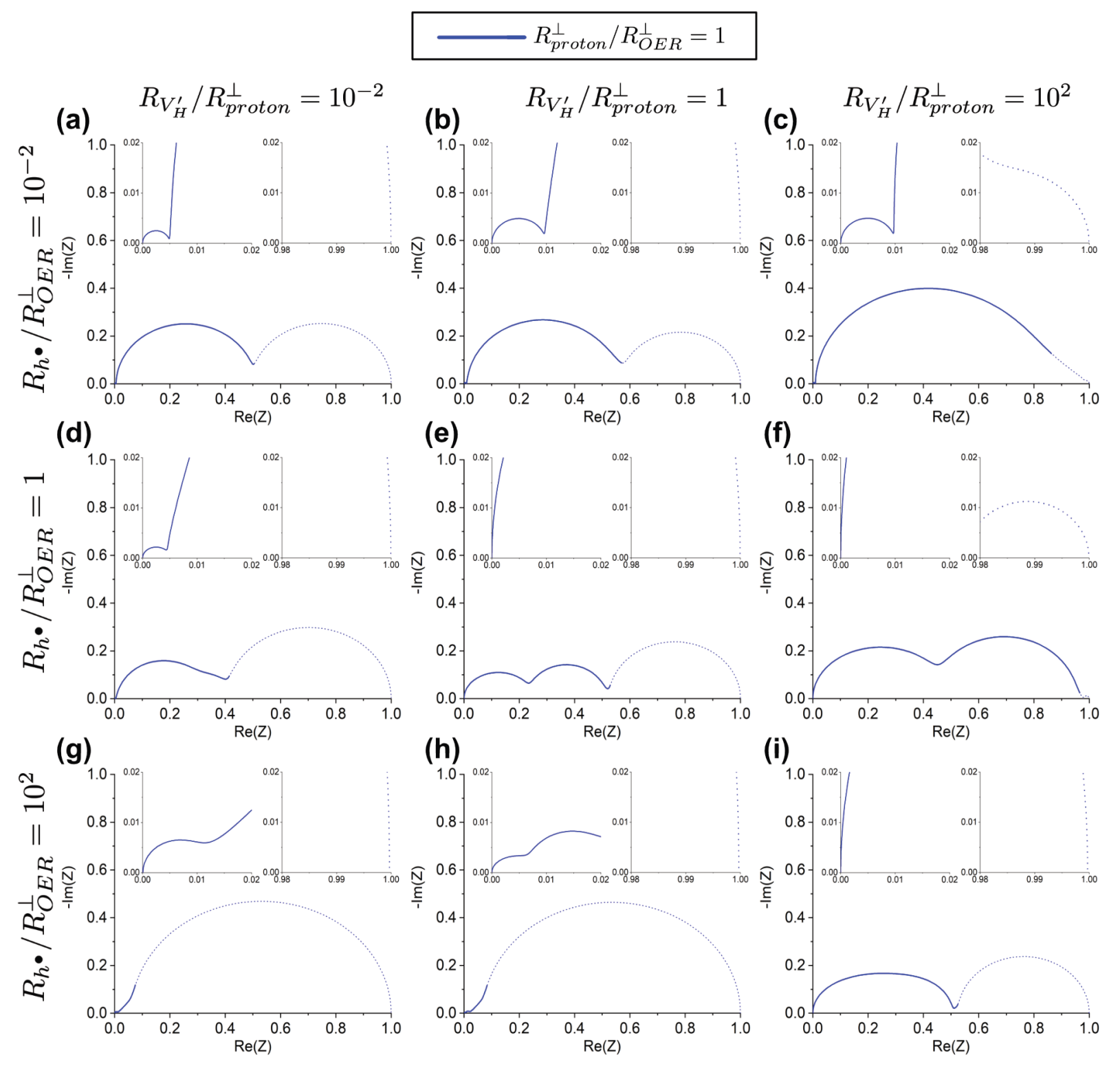

Figure A-1. Calculated Nyquist plots with various input parameters. Same inputs as Figure 5, except $R_{\text {proton }}^{\perp} / R_{O E R}^{\perp}=1$. 


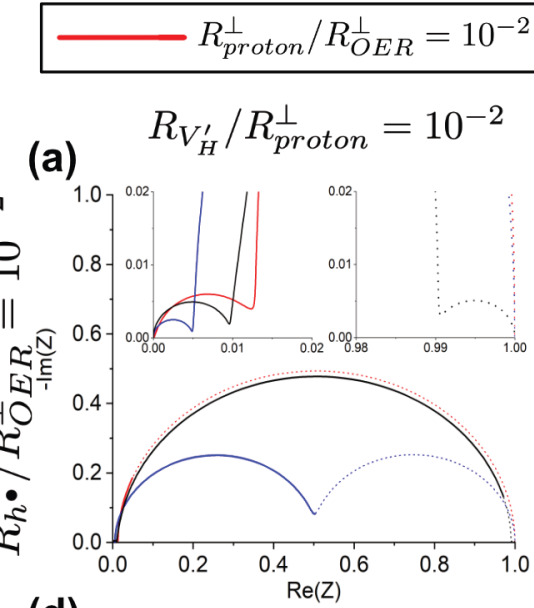

(d)

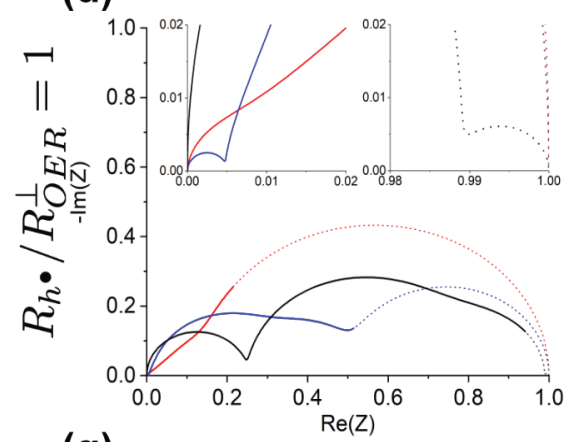

(g)

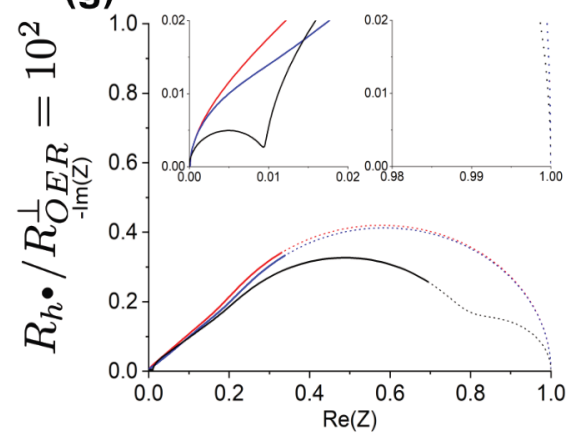

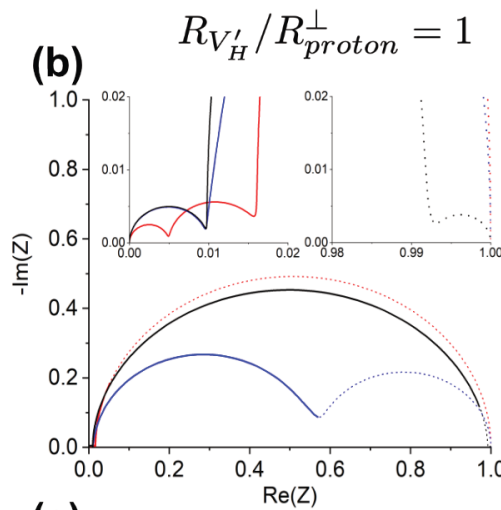

(e)

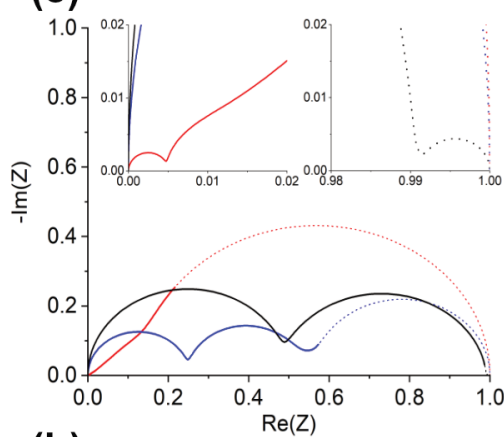

(h)

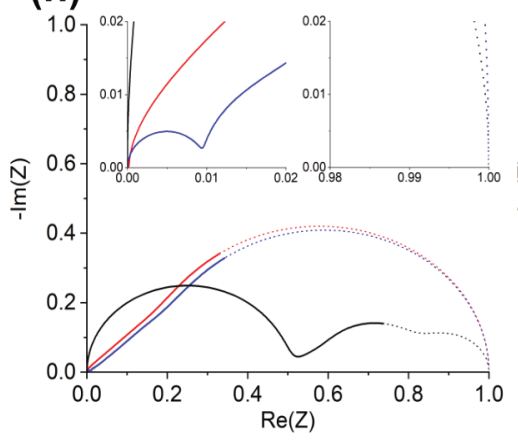

$R_{\text {proton }}^{\perp} / R_{O E R}^{\perp}=10^{2}$

(c) $R_{V_{H}^{\prime}} / R_{\text {proton }}^{\perp}=10^{2}$

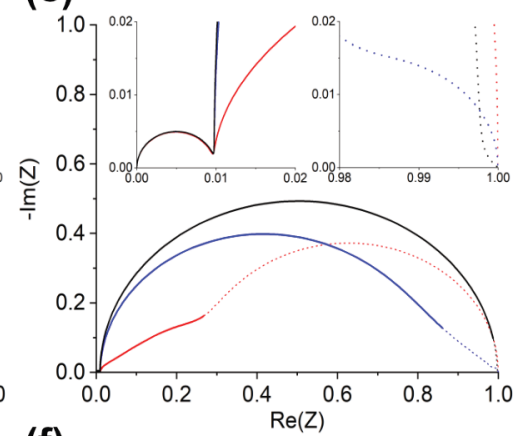

(f)

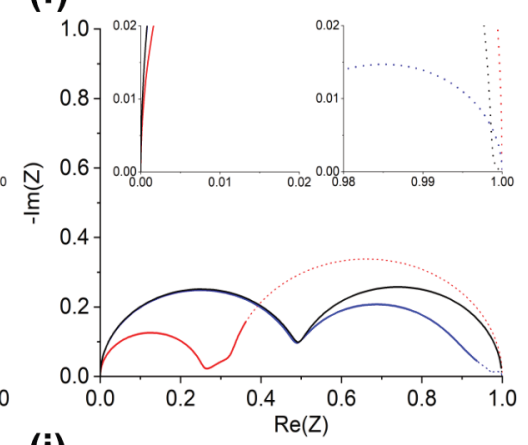

(i)

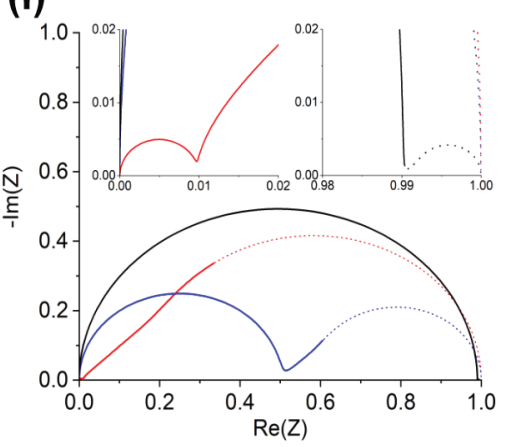

Figure A-2. Calculated Nyquist plots with various input parameters and no DC bias applied. $C_{c h e m} / C_{d l}$ is fixed at 1000 . 


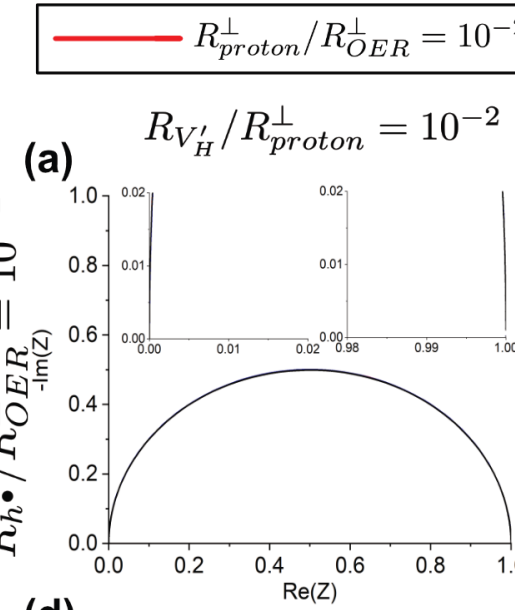

\section{(d)}

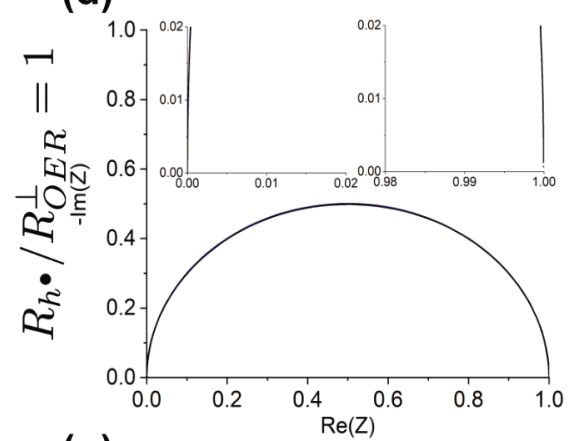

(g)

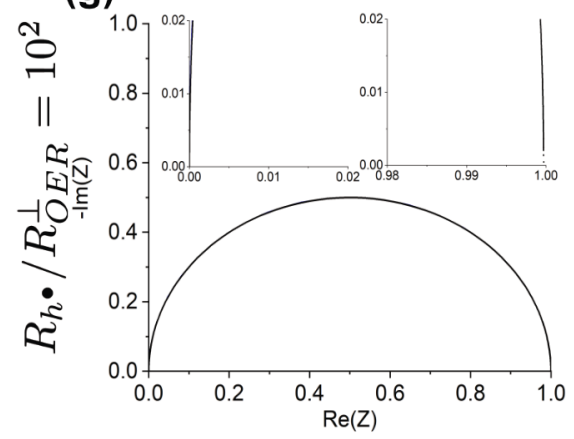

(b)

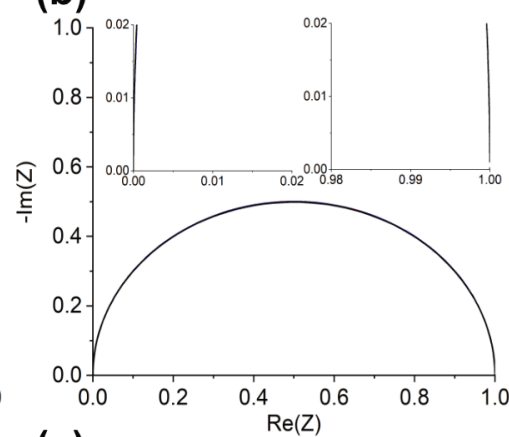

(e)

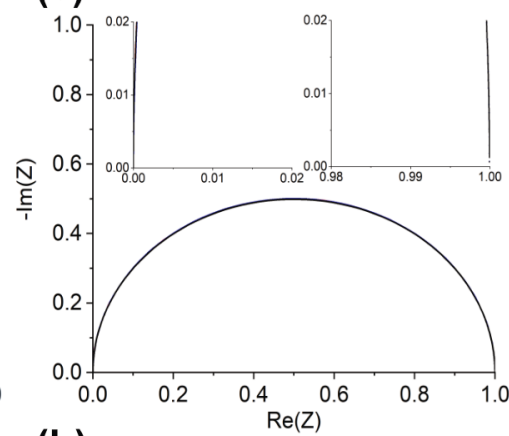

(h)

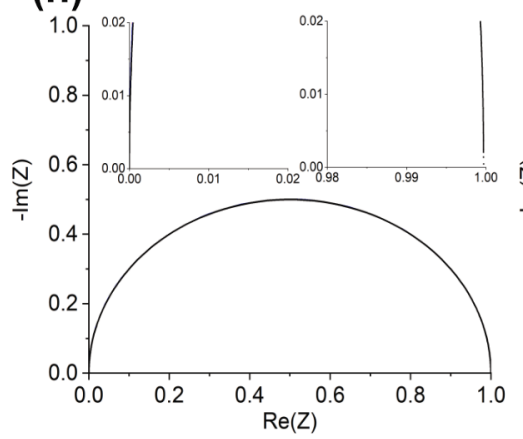

$R_{\text {proton }}^{\perp} / R_{O E R}^{\perp}=10^{2}$

$R_{V_{H}^{\prime}} / R_{\text {proton }}^{\perp}=10^{2}$

(c)

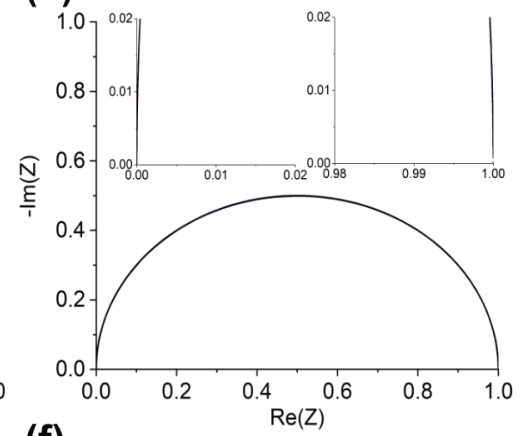

(f)

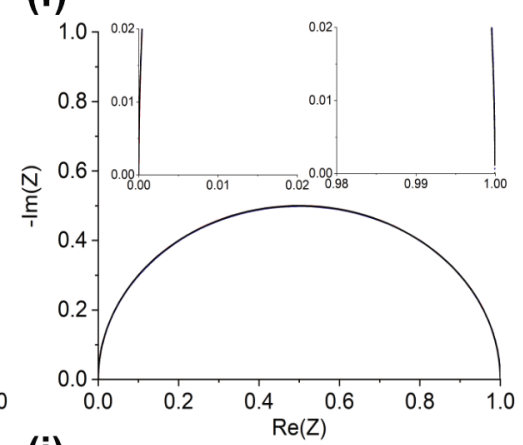

(i)

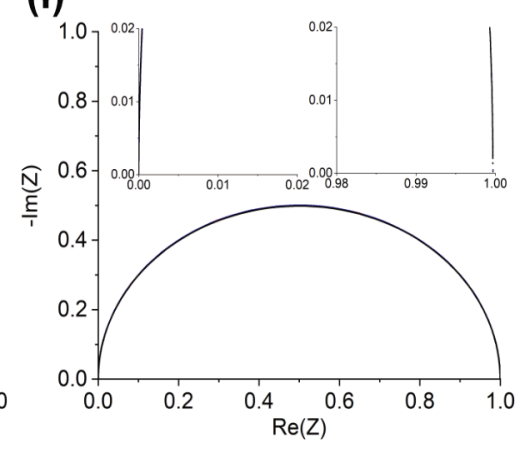

Figure A-3. Calculated Nyquist plots with various input parameters and applied DC bias. $C_{c h e m} / C_{d l}$ is fixed at 0.001 . 


\begin{tabular}{|c|c|c|c|c|c|c|}
\hline & \multicolumn{2}{|c|}{$\begin{array}{c}R_{\text {proton }}^{\perp} / R_{O E R}^{\perp}=0.01 \\
\text { Figure 5 }\end{array}$} & \multicolumn{2}{|c|}{$\begin{array}{c}R_{\text {proton }}^{\perp} / R_{O E R}^{\perp}=100 \\
\text { Figure } 6\end{array}$} & \multicolumn{2}{|c|}{$\begin{array}{c}R_{\text {proton }}^{\perp} / R_{O E R}^{\perp}=1 \\
\text { Figure A-1 }\end{array}$} \\
\hline & $\boldsymbol{R}_{V_{H}^{\prime}}$ & $\boldsymbol{R}_{h^{\bullet}}$ & $\boldsymbol{R}_{V_{H}^{\prime}}$ & $\boldsymbol{R}_{\boldsymbol{h}^{\bullet}}$ & $\boldsymbol{R}_{V_{H}^{\prime}}$ & $R_{h^{\bullet}}$ \\
\hline $\mathbf{a}$ & $9.90 \times 10^{-5}$ & 0.0099 & 0.99 & 0.0099 & 0.0099 & 0.0099 \\
\hline b & 0.0099 & 0.0099 & 99.01 & 0.0099 & 0.99 & 0.0099 \\
\hline c & 0.99 & 0.0099 & 9901 & 0.0099 & 99.01 & 0.0099 \\
\hline d & $5.00 \times 10^{-5}$ & 0.5 & 0.5 & 0.5 & 0.005 & 0.5 \\
\hline $\mathbf{e}$ & 0.005 & 0.5 & 50 & 0.5 & 0.5 & 0.5 \\
\hline f & 0.5 & 0.5 & 5000 & 0.5 & 50 & 0.5 \\
\hline g & $9.90 \times 10^{-7}$ & 0.99 & 0.0099 & 0.99 & $9.90 \times 10^{-5}$ & 0.99 \\
\hline $\mathbf{h}$ & $9.90 \times 10^{-5}$ & 0.99 & 0.99 & 0.99 & 0.0099 & 0.99 \\
\hline i & 0.0099 & 0.99 & 99.01 & 0.99 & 0.99 & 0.99 \\
\hline
\end{tabular}

Table A-1. Normalized input values for $R_{V_{H}^{\prime}}$ and $R_{h}$ • in calculation of the Nyquist plots in Figure 5-6 and Figure A-1. Note that, with the normalization method chosen in this study, $R_{O E R}^{\perp}=1-R_{h^{\circ}}$. 


\begin{tabular}{|c|c|c|c|c|c|c|}
\hline & \multicolumn{2}{|c|}{$\begin{array}{c}R_{\text {proton }}^{\perp} / R_{O E R}^{\perp}=0.01 \\
\text { Figure } 5\end{array}$} & \multicolumn{2}{|c|}{$\begin{array}{c}R_{\text {proton }}^{\perp} / R_{O E R}^{\perp}=100 \\
\text { Figure } 6\end{array}$} & \multicolumn{2}{|c|}{$\begin{array}{c}R_{\text {proton }}^{\perp} / R_{O E R}^{\perp}=1 \\
\text { Figure A-1 }\end{array}$} \\
\hline & $R_{1}^{a r c}$ & $R_{2}^{a r c}$ & $R_{1}^{a r c}$ & $R_{2}^{a r c}$ & $R_{1}^{a r c}$ & $R_{2}^{a r c}$ \\
\hline $\mathbf{a}$ & $9.80 \times 10^{-5}$ & 0.0098 & 0.0098 & 0.98 & 0.005 & 0.5 \\
\hline $\mathbf{b}$ & 0.0050 & 0.0098 & 0.0099 & 0.98 & 0.0098 & 0.5 \\
\hline c & 0.0099 & 0.0098 & 0.0099 & 0.98 & 0.0099 & 0.5 \\
\hline d & $5.00 \times 10^{-5}$ & 0.005 & 0.25 & 0.5 & 0.005 & 0.25 \\
\hline $\mathbf{e}$ & 0.005 & 0.005 & 0.495 & 0.5 & 0.25 & 0.25 \\
\hline $\mathbf{f}$ & 0.25 & 0.005 & 0.5 & 0.5 & 0.5 & 0.25 \\
\hline g & $9.90 \times 10^{-7}$ & $9.80 \times 10^{-5}$ & 0.0098 & 0.0098 & $9.90 \times 10^{-5}$ & 0.005 \\
\hline $\mathbf{h}$ & $9.90 \times 10^{-5}$ & $9.80 \times 10^{-5}$ & 0.495 & 0.0098 & 0.0098 & 0.005 \\
\hline $\mathbf{i}$ & 0.0098 & $9.80 \times 10^{-5}$ & 0.98 & 0.0098 & 0.495 & 0.005 \\
\hline
\end{tabular}

Table A-2. Expected values of $R_{1}^{\text {arc }}$ and $R_{2}^{\text {arc }}$ of the Nyquist plots in Figure 5-6 and Figure A-1. 


\section{References}

1. V. Augustyn, P. Simon, and B. Dunn, Energ Environ Sci, 7, 1597 (2014).

2. R. P. Forslund, J. Pender, C. T. Alexander, K. P. Johnston, and K. J. Stevenson, J Mater Chem A, 7, 21222 (2019).

3. J. T. Mefford, W. G. Hardin, S. Dai, K. P. Johnston, and K. J. Stevenson, Nat Mater, 13, 726 (2014).

4. W. Sugimoto, H. Iwata, K. Yokoshima, Y. Murakami, and Y. Takasu, J Phys Chem B, 109, 7330 (2005).

5. M. Salanne, B. Rotenberg, K. Naoi, K. Kaneko, P.-L. Taberna, C. P. Grey, B. Dunn, and P. Simon, Nat Energy, 1, 1 (2016).

6. Y. Shao, M. F. El-Kady, J. Sun, Y. Li, Q. Zhang, M. Zhu, H. Wang, B. Dunn, and R. B. Kaner, Chem Rev, 118, 9233 (2018).

7. M. R. Lukatskaya, B. Dunn, and Y. Gogotsi, Nat Commun, 7, 12647 (2016).

8. J. T. Mefford, X. Rong, A. M. Abakumov, W. G. Hardin, S. Dai, A. M. Kolpak, K. P. Johnston, and K. J. Stevenson, Nat Commun, 7, 1 (2016).

9. R. P. Forslund, W. G. Hardin, X. Rong, A. M. Abakumov, D. Filimonov, C. T. Alexander, J. T. Mefford, H. Iyer, A. M. Kolpak, K. P. Johnston, and K. J. Stevenson, Nat Commun, 9, 3150 (2018).

10. M. S. Burke, L. J. Enman, A. S. Batchellor, S. Zou, and S. W. Boettcher, Chem Mater, 27, 7549 (2015).

11. A. Grimaud, O. Diaz-Morales, B. Han, W. T. Hong, Y.-L. Lee, L. Giordano, K. A. Stoerzinger, M. T. M. Koper, and Y. Shao-Horn, Nat Chem, 9, 457 (2017).

12. R. L. Doyle, I. J. Godwin, M. P. Brandon, and M. E. G. Lyons, Phys Chem Chem Phys, 15, 13737 (2013).

13. C. Sun, R. Hui, and J. Roller, J Solid State Electr, 14, 1125 (2010).

14. Z. A. Feng, F. E. Gabaly, X. Ye, Z.-X. Shen, and W. C. Chueh, Nat Commun, 5, 4374 (2014).

15. A. B. Stambouli and E. Traversa, Renew Sustain Energy Rev, 6, 433 (2002).

16. Y. Li and W. C. Chueh, Annu Rev Mater Res, 48, 137 (2018).

17. J. Jamnik, Solid State Ionics, 157, 19 (2003).

18. J. Jamnik and J. Maier, Phys Chem Chem Phys, 3, 1668 (2001).

19. J. Jamnik and J. Maier, J Electrochem Soc, 146, 4183 (1999).

20. R. L. Doyle and M. E. G. Lyons, Phys Chem Chem Phys, 15, 5224 (2013).

21. M. E. G. Lyons and M. P. Brandon, J Electroanal Chem, 631, 62 (2009).

22. B. D. Cahan and C. T. Chen, J Electrochem Soc, 129, 700 (1982).

23. F. Ciucci, Curr Opin Electrochem, 13, 132 (2018).

24. R. H. Nilson, M. T. Brumbach, and B. C. Bunker, J Electrochem Soc, 158, A678 (2011).

25. W. Lai and S. M. Haile, Phys Chem Chem Phys, 10, 865 (2008).

26. W. Lai and S. M. Haile, J Am Ceram Soc, 88, 2979 (2005).

27. S. B. Adler, Chem Rev, 104, 4791 (2004).

28. B. A. Boukamp and H. J. M. Bouwmeester, Solid State Ionics, 157, 29 (2003).

29. B. A. Boukamp, Solid State Ionics, 169, 65 (2004).

30. W. Lai and F. Ciucci, Electrochim Acta, 56, 531 (2010).

31. E. Quattrocchi, T. H. Wan, A. Curcio, S. Pepe, M. B. Effat, and F. Ciucci, Electrochim Acta, 324, 134853 (2019).

32. D. Gruet, B. Delobel, D. Sicsic, I. T. Lucas, and V. Vivier, Electrochim Acta, 295, 787 (2018).

33. G. Sikha and R. E. White, J Electrochem Soc, 155, A893 (2008).

34. M. Doyle, J. P. Meyers, and J. Newman, J Electrochem Soc, 147, 99 (2000).

35. J. P. Meyers, M. Doyle, R. M. Darling, and J. Newman, J Electrochem Soc, 147, 2930 (2000).

36. M. H. Hebb, J Chem Phys, 20, 185 (1952).

37. C. Wagner, Zeitschrift Für Elektrochemie Berichte Der Bunsengesellschaft Für Physikalische Chemie, 60, 4 (1956).

38. I. Riess, Solid State Ionics, 91, 221 (1996).

39. D. A. Corrigan, J Electrochem Soc, 134, 377 (1987).

40. D. A. Corrigan and R. M. Bendert, J Electrochem Soc, 136, 723 (1989).

41. R. D. Armstrong and E. A. Charles, J Power Sources, 25, 89 (1989).

42. M. B. Stevens, L. J. Enman, A. S. Batchellor, M. R. Cosby, A. E. Vise, C. D. M. Trang, and S. W. Boettcher, Chem Mater, 29, 120 (2016).

43. M. S. Burke, S. Zou, L. J. Enman, J. E. Kellon, C. A. Gabor, E. Pledger, and S. W. Boettcher, J Phys Chem Lett, 6, 3737 (2015).

44. R. Barnard, C. F. Randell, and F. L. Tye, J Appl Electrochem, 10, 109 (1980).

45. P. Oliva, J. Leonardi, J. F. Laurent, C. Delmas, J. J. Braconnier, M. Figlarz, F. Fievet, and A. de Guibert, J Power Sources, 8, 229 (1982).

46. V. Mancier, A. Métrot, and P. Willmann, Electrochim Acta, 41, 1259 (1996).

47. S. Motupally, C. C. Streinz, and J. W. Weidner, J Electrochem Soc, 142, 1401 (1995).

48. I. R. Tucceri, International Journal of Advances in Chemnistry, 2, 23 (2016).

49. V. Srinivasan, J. W. Weidner, and R. E. White, J Solid State Electr, 4, 367 (2000).

50. J. R. Macdonald, J Electroanal Chem Interfacial Electrochem, 223, 25 (1987).

51. E. Barsoukov and Dr. J. R. Macdonald, Impedance Spectroscopy, John Wiley \& Sons, Inc., Hoboken, NJ, (2005).

52. F. Wohde, M. Balabajew, and B. Roling, J Electrochem Soc, 163, A714 (2016). 
53. Y.-F. Li and A. Selloni, Acs Catal, 4, 1148 (2014).

54. V. Fidelsky and M. C. Toroker, Phys Chem Chem Phys, 19, 7491 (2017).

55. F. A. L. Laskowski, M. R. Nellist, J. Qiu, and S. W. Boettcher, J Am Chem Soc, 141, 1394 (2018).

56. M. Ikoma, Y. Hoshina, I. Matsumoto, and C. Iwakura, J Electrochem Soc, 143, 1904 (1996).

57. F. Bardé, M. R. Palacín, B. Beaudoin, P. A. Christian, and J.-M. Tarascon, J Power Sources, 160, 733 (2006).

58. P. L. Bourgault and B. E. Conway, Can J Chem, 38, 1557 (1960).

59. B. E. Conway and P. L. Bourgault, Can J Chem, 37, 292 (1959).

60. H. Dotan, A. Landman, S. W. Sheehan, K. D. Malviya, G. E. Shter, D. A. Grave, Z. Arzi, N. Yehudai, M. Halabi, N. Gal, N. Hadari, C. Cohen, A. Rothschild, and G. S. Grader, Nat Energy, 4, 786 (2019).

61. J. Rajeswari, P. Kishore, B. Viswanathan, and T. Varadarajan, Nanoscale Res Lett, 2, 496 (2007).

62. H. Razmi and R. Mohammad-Rezaei, Electrochim Acta, 56, 7220 (2011).

63. Y. L. Cao, H. X. Yang, X. P. Ai, and L. F. Xiao, J Electroanal Chem, 557, 127 (2003).

64. C. Yang, G. Rousse, K. L. Svane, P. E. Pearce, A. M. Abakumov, M. Deschamps, G. Cibin, A. V. Chadwick, D. A. D. Corte, H. A. Hansen, T. Vegge, J.-M. Tarascon, and A. Grimaud, Nat Commun, 11, 1378 (2020).

65. T. R. Brumlev and R. P. Buck, J Electroanal Chem Interfacial Electrochem, 126, 73 (1981).

66. G. C. Barker, J Electroanal Chem Interfacial Electrochem, 41, 201 (1973).

67. Z. Chen, J Electrochem Soc, 151, A1576 (2004).

68. M. Kato, J Theor Biol, 177, 299 (1995).

69. W. Li, B. Guan, T. Yang, N. Zhang, X. Zhang, and X. Liu, Phys Chem Chem Phys, 19, 23218 (2017).

70. A. J. Bard and L. R. Faulkner, Electrochemical Methods: Fundamentals and Applications, 2nd Edition, John Wiley \& Sons, Inc., (2000).

71. C. Ho, I. D. Raistrick, and R. A. Huggins, J Electrochem Soc, 127, 343 (1980). 